\title{
Positive Solutions for Anisotropic Singular Dirichlet Problems
}

\author{
Nikolaos S. Papageorgiou ${ }^{1} \cdot$ Andrea Scapellato $^{2}$ \\ Received: 9 September 2021 / Revised: 31 October 2021 / Accepted: 17 January 2022 / \\ Published online: 25 February 2022 \\ (c) The Author(s) 2022
}

\begin{abstract}
We consider a Dirichlet problem driven by a $(p(z), q(z))$-Laplacian and a reaction involving the sum of a parametric singular term plus a superlinear perturbation. We prove a bifurcation-type result describing the changes in the set of positive solutions as the parameter $\lambda>0$ varies. Also we show that for every admissible parameter the problem has a smallest positive solution and obtain the monotonicity and continuity properties of the minimal solution map.
\end{abstract}

Keywords Regularity theory · Maximum principle $\cdot$ Truncation · Positive solution · Minimal solution · Hardy's inequality

Mathematics Subject Classification 35J60 - 35J92

\section{Introduction}

In this paper, we study the following anisotropic singular Dirichlet problem

Communicated by Rosihan M. Ali.

$凶$ Andrea Scapellato

andrea.scapellato@unict.it

Nikolaos S. Papageorgiou

npapg@math.ntua.gr

1 Department of Mathematics, National Technical University, Zografou Campus, 15780 Athens, Greece

2 Dipartimento di Matematica e Informatica, Università degli Studi di Catania, Viale Andrea Doria 6, 95125 Catania, Italy 


$$
\left\{\begin{array}{l}
-\Delta_{p(z)} u(z)-\Delta_{q(z)} u(z)=\lambda u(z)^{-\eta(z)}+f(z, u(z)) \text { in } \Omega \\
\left.u\right|_{\partial \Omega}=0, u>0, \lambda>0
\end{array} .\right.
$$

In this problem, $\Omega \subseteq \mathbb{R}^{N}$ is a bounded domain with a $C^{2}$-boundary $\partial \Omega$. If $E_{1}=$ $\left\{r \in C(\bar{\Omega}): 1<\min _{\bar{\Omega}} r\right\}$, then for $r \in E_{1}$, by $\Delta_{r(z)}$ we denote the anisotropic $r$-Laplace differential operator defined by

$$
\Delta_{r(z)}=\operatorname{div}\left(|D u|^{r(z)-2} D u\right) \text { for all } u \in W_{0}^{1, r(z)}(\Omega) .
$$

In contrast to the isotropic $r$-Laplacian (that is, $r(\cdot)$ is constant), the anisotropic one is nonhomogeneous. In $\left(\mathrm{P}_{\lambda}\right)$ we have the sum of two such operators. So, even in the isotropic case, the differential operator of $\left(\mathrm{P}_{\lambda}\right)$ is not homogeneous and this makes the study of $\left(\mathrm{P}_{\lambda}\right)$ more difficult. In the reaction [right-hand side of $\left(\mathrm{P}_{\lambda}\right)$ ], we have the combined effects of two nonlinear terms of different nature. One is the parametric singular term $u \mapsto \lambda u^{-\eta(z)}$ with $\lambda>0$ being the parameter, with $\eta \in$ $C(\bar{\Omega})$ and $0<\eta_{-}=\min _{\bar{\Omega}} \eta \leq \max _{\bar{\Omega}} \eta=\eta_{+}<1$. The other is a Carathéodory perturbation $f(z, x)$ (that is, for all $x \in \mathbb{R}, z \mapsto f(z, x)$ is measurable and for a.a. $z \in \Omega, x \mapsto f(z, x)$ is continuous). We assume that for a.a. $z \in \Omega, f(z, \cdot)$ is $\left(p_{+}-1\right)$-superlinear $\left(p_{+}=\max _{\bar{\Omega}} p\right)$, but need not satisfy the usual in such cases Ambrosetti-Rabinowitz condition (the AR-condition for short). We search for the existence of positive solutions, and our goal is to produce a precise description of the changes in the set of positive solutions as the parameter $\lambda$ varies in the open semiaxis $\stackrel{\circ}{\mathbb{R}}_{+}=(0,+\infty)$.

Anisotropic boundary value problems have been studied extensively in the last decade. We refer to the books of Diening-Harjulehto-Hästo-Růžička [4] and Rădulescu-Repovš [19] and the references therein. The study of singular anisotropic problems is lagging behind. Very few works on the subject can be found in the literature. We mention two recent ones by Byun-Ko [2] and Saoudi-Ghanmi [22] which are closely related to our work here. Both papers deal with equations driven by the Dirichlet anisotropic $p$-Laplacian and their hypotheses on the data are more restrictive (see hypothesis $\left(p_{M}\right)$ in Byun-Ko [2] and hypotheses $\left(\mathrm{H}_{1}\right)-\left(\mathrm{H}_{4}\right)$ in Saoudi-Ghanmi [22]). In addition, our approach is different.

A main difficulty that we encounter when we deal with singular problems is that the corresponding energy (Euler) functional is not $C^{1}$, and so we cannot use the results of critical point theory directly on it. Therefore, we need to find a way to isolate the singularity and deal with $C^{1}$ functionals. For this reason, first we study an auxiliary purely singular problem for which we prove an existence and uniqueness result. Using this solution we are able to bypass the singularity and then, using variational tools from the critical point theory together with truncation and comparison techniques, we prove a bifurcation-type theorem describing the changes in the set of positive solutions of $\left(\mathrm{P}_{\lambda}\right)$. According to our theorem, there exists a critical parameter $\lambda^{*}>0$ such that

- for all $\lambda \in\left(0, \lambda^{*}\right)$ problem $\left(\mathrm{P}_{\lambda}\right)$ has at least two positive smooth solutions; 
- for $\lambda=\lambda^{*}$ problem $\left(\mathrm{P}_{\lambda}\right)$ has at least one positive smooth solution;

- for all $\lambda>\lambda^{*}$ problem $\left(\mathrm{P}_{\lambda}\right)$ has no positive solutions.

Moreover, we show that for every admissible parameter $\lambda \in\left(0, \lambda^{*}\right]$, problem $\left(\mathrm{P}_{\lambda}\right)$ has a smallest positive solution $u_{\lambda}^{*}$ (minimal or barrier solution) and we establish the monotonicity and continuity properties of the map $\lambda \mapsto u_{\lambda}^{*}$.

For further details on the study of singular equations, we refer the reader to the papers [20, 21, 23].

\section{Mathematical Background: Hypotheses}

The study of problem $\left(\mathrm{P}_{\lambda}\right)$ requires the use of Lebesgue and Sobolev spaces with variable exponents. A comprehensive presentation of the theory of these spaces can be found in the book of Diening-Harjuletho-Hästö-Rǔžička [4].

Let $M(\Omega)$ be the vector space of all measurable functions from $\Omega$ into $\mathbb{R}$. We identify two such functions which differ only on a Lebesgue-null set. Given $r \in E_{1}$, the anisotropic Lebesgue space $L^{r(z)}(\Omega)$ is defined by

$$
L^{r(z)}(\Omega)=\left\{u \in M(\Omega): \int_{\Omega}|u|^{r(z)} \mathrm{d} z<\infty\right\} .
$$

We equip this space with the so-called Luxemburg norm defined by

$$
\|u\|_{r(z)}=\inf \left[\vartheta>0: \int_{\Omega}\left(\frac{|u(z)|}{\vartheta}\right)^{r(z)} \mathrm{d} z \leq 1\right] .
$$

Closely related to this norm is the modular function $\rho_{r}(\cdot)$ defined by

$$
\rho_{r}(u)=\int_{\Omega}|u|^{r(z)} \mathrm{d} z \quad \text { for all } u \in L^{r(z)}(\Omega) .
$$

Evidently $\|\cdot\|_{r(z)}$ is the Minkowski functional of the set

$$
C=\left\{u \in L^{r(z)}(\Omega): \rho_{r}(u) \leq 1\right\}
$$

Also, we have the following proposition which illustrates the close relation between $\|\cdot\|_{r(z)}$ and $\rho_{r}(\cdot)$. Recall that, if $r \in E_{1}$, then $r_{-}=\min _{\bar{\Omega}} r, r_{+}=\max _{\bar{\Omega}} r$.

Proposition 2.1 If $r \in E_{1}$ and $\left\{u_{n}, u\right\}_{n \in \mathbb{N}} \subseteq L^{r(z)}(\Omega)$, then

(a) $\|u\|_{r(z)}=\vartheta \Leftrightarrow \rho_{r}\left(\frac{u}{\vartheta}\right)=1$;

(b) $\|u\|_{r(z)}<1($ resp. $=1,>1) \Leftrightarrow \rho_{r}(u)<1($ resp. $=1,>1)$;

(c) $\|u\|_{r(z)} \leq 1 \Rightarrow\|u\|_{r(z)}^{r_{+}} \leq \rho_{r}(u) \leq\|u\|_{r(z)}^{r_{-}}$;

$\|u\|_{r(z)} \geq 1 \Rightarrow\|u\|_{r(z)}^{r_{-}} \leq \rho_{r}(u) \leq\|u\|_{r(z)}^{r_{+}}$;

(d) $\left\|u_{n}\right\|_{r(z)} \rightarrow 0$ (resp. $\left.\rightarrow \infty\right) \Leftrightarrow \rho_{r}\left(u_{n}\right) \rightarrow 0($ resp. $\rightarrow \infty)$; 
(e) $\left\|u_{n}-u\right\|_{r(z)} \rightarrow 0 \Leftrightarrow \rho_{r}\left(u_{n}-u\right) \rightarrow 0$.

The space $L^{r(z)}(\Omega)\left(r \in E_{1}\right)$ is a Banach space which is separable, reflexive (in fact uniformly convex). Also, if $r^{\prime} \in E_{1}$ is given by $r^{\prime}(z)=\frac{r(z)}{r(z)-1}$ for all $z \in \bar{\Omega}$ (that is, $\frac{1}{r(z)}+\frac{1}{r^{\prime}(z)}=1$ for all $\left.z \in \bar{\Omega}\right)$, then

$$
L^{r(z)}(\Omega)^{*}=L^{r^{\prime}(z)}(\Omega)
$$

Moreover, we have the following version of Hölder's inequality

$$
\int_{\Omega}|u v| \mathrm{d} z \leq\left(\frac{1}{r_{-}}+\frac{1}{r_{-}^{\prime}}\right)\|u\|_{r(z)}\|v\|_{r^{\prime}(z)} \text { for all } u \in L^{r(z)}(\Omega), \text { all } v \in L^{r^{\prime}(z)}(\Omega) .
$$

We know that, if $q, r \in E_{1}$ and $q(z) \leq r(z)$ for all $z \in \bar{\Omega}$, then

$$
L^{r(z)}(\Omega) \hookrightarrow L^{q(z)}(\Omega) \hookrightarrow L^{1}(\Omega) \text { continuously. }
$$

Using the anisotropic Lebesgue spaces, we can define also anisotropic Sobolev spaces.

Given $r \in E_{1}$ we define

$$
W^{1, r(z)}(\Omega)=\left\{u \in L^{r(z)}(\Omega):|D u| \in L^{r(z)}(\Omega)\right\} .
$$

We equip this space with the following norm:

$$
\|u\|_{1, r(z)}=\|u\|_{r(z)}+\|D u\|_{r(z)} .
$$

Here $\|D u\|_{r(z)}=\||D u|\|_{r(z)}$. Suppose that $r \in E_{1} \cap C^{0,1}(\bar{\Omega})$ (that is, assume that the variable exponent is Lipschitz continuous). We define

$$
W_{0}^{1, r(z)}(\Omega)={\overline{C_{c}^{\infty}(\Omega)}}^{\|\cdot\|_{1, r(z)}} .
$$

Both spaces $W^{1, r(z)}(\Omega)$ and $W_{0}^{1, r(z)}(\Omega)$ are Banach spaces which are separable and reflexive (in fact uniformly convex), Moreover, for the space $W_{0}^{1, r(z)}(\Omega)$ the Poincare inequality holds, namely

$$
\|u\|_{r(z)} \leq \widehat{c}\|D u\|_{r(z)} \text { for some } \widehat{c}>0, \text { all } u \in W_{0}^{1, r(z)}(\Omega)
$$

If $r \in E_{1} \cap C^{0,1}(\bar{\Omega})$, then we define

$$
r^{*}(z)=\left\{\begin{array}{ll}
\frac{N r(z)}{N-r(z)} & \text { if } r(z)<N \\
+\infty & \text { if } N \leq r(z)
\end{array} \text { for all } z \in \bar{\Omega}\right.
$$


This is the variable critical Sobolev exponent corresponding to $r(\cdot)$. Suppose $q \in$ $C(\bar{\Omega})$ and assume that

$$
1 \leq q_{-} \leq q(z) \leq r^{*}(z) \quad\left(\text { resp. } 1 \leq q_{-} \leq q(z)<r^{*}(z)\right) \text { for all } z \in \bar{\Omega}
$$

Let $X=W^{1, r(z)}(\Omega)$ or $X=W_{0}^{1, r(z)}(\Omega)$. Then, we have that $X \hookrightarrow L^{q(z)}(\Omega)$ continuously (resp. $X \hookrightarrow L^{q(z)}(\Omega)$ compactly). This is the so-called anisotropic Sobolev embedding theorem. For $r \in E_{1} \cap C^{0,1}(\bar{\Omega})$, we have

$$
W_{0}^{1, r(z)}(\Omega)^{*}=W^{-1, r^{\prime}(z)}(\Omega)
$$

Consider the nonlinear operator $A_{r(z)}: W_{0}^{1, r(z)}(\Omega) \rightarrow W^{-1, r^{\prime}(z)}(\Omega)$ defined by

$$
\left\langle A_{r(z)}(u), h\right\rangle=\int_{\Omega}|D u|^{r(z)-2}(D u, D h)_{\mathbb{R}^{N}} \mathrm{~d} z \text { for all } u, h \in W_{0}^{1, r(z)}(\Omega) .
$$

This operator has the following properties (see Gasiński-Papageorgiou [8], Proposition 2.5 and Rădulescu-Repovš [19], p. 40).

Proposition 2.2 The operator $A_{r(z)}(\cdot)$ is bounded (maps bounded sets to bounded sets), continuous, strictly monotone (hence maximal monotone too) and of type $(S)_{+}$, that is, it has the following property

$$
\begin{aligned}
& \text { "If } u_{n} \stackrel{w}{\rightarrow} u \text { in } W_{0}^{1, r(z)}(\Omega) \text { and } \limsup _{n \rightarrow \infty}\left\langle A_{r(z)}\left(u_{n}\right), u_{n}-u\right\rangle \leq 0, \\
& \text { then } u_{n} \rightarrow u \text { in } W_{0}^{1, r(z)}(\Omega) \text { as } n \rightarrow \infty " .
\end{aligned}
$$

Another space that we will use (as a result of the anisotropic regularity theory) is the space $C_{0}^{1}(\bar{\Omega})=\left\{u \in C^{1}(\bar{\Omega}):\left.u\right|_{\partial \Omega}=0\right\}$. This is an ordered Banach space with positive (order) cone $C_{+}=\left\{u \in C_{0}^{1}(\bar{\Omega}): u(z) \geq 0\right.$ for all $\left.z \in \bar{\Omega}\right\}$. This cone has a nonempty interior given by

$$
\text { int } C_{+}=\left\{u \in C_{+}: u(z)>0 \text { for all } z \in \Omega,\left.\frac{\partial u}{\partial n}\right|_{\partial \Omega}<0\right\} \text {, }
$$

with $n(\cdot)$ being the outward unit normal on $\partial \Omega$.

Our hypotheses on the exponents involved in problem $\left(\mathrm{P}_{\lambda}\right)$ are the following:

$\mathrm{H}_{0}: p, q \in C^{0,1}(\bar{\Omega}), \eta \in C(\bar{\Omega}), q_{-} \leq q_{+}<p_{-} \leq p_{+}$and $0<\eta(z)<1$ for all $z \in \bar{\Omega}$.

Consider $h_{1}, h_{2} \in M(\Omega)$. We write $h_{1} \preceq h_{2}$ if and only if for every $K \subseteq \Omega$ compact we have $0<c_{K} \leq h_{2}(z)-h_{1}(z)$ for a.a. $z \in K$. Note that if $h_{1}, h_{2} \in C(\Omega)$ and $h_{1}(z)<h_{2}(z)$ for all $z \in \Omega$, then $h_{1} \preceq h_{2}$. Using this ordering notion and following the argument in the proof of Proposition 2.4 of Papageorgiou-Rădulescu-Repovš [17], we obtain the following strong comparison theorem for singular problems. 
Proposition 2.3 If hypotheses $\mathrm{H}_{0}$ hold, $\widehat{\xi} \in L^{\infty}(\Omega), \widehat{\xi}(z) \geq 0$ for a.a. $z \in \Omega, h_{1}, h_{2} \in$ $L^{\infty}(\Omega), h_{1} \preceq h_{2}, u \in W_{0}^{1, p(z)}(\Omega), u \geq 0, u \neq 0$ and $v \in \operatorname{int} C_{+}$satisfy

$$
\begin{aligned}
& -\Delta_{p(z)} u-\Delta_{q(z)} u+\widehat{\xi}(z) u^{p(z)-1}-\lambda u^{-\eta(z)}=h_{1} \text { in } \Omega, \\
& -\Delta_{p(z)} v-\Delta_{q(z)} v+\widehat{\xi}(z) v^{p(z)-1}-\lambda v^{-\eta(z)}=h_{2} \quad \text { in } \Omega,\left.\quad \frac{\partial v}{\partial n}\right|_{\partial \Omega}<0,
\end{aligned}
$$

then $v-u \in \operatorname{int} C_{+}$.

For every $u \in M(\Omega)$, we set $u^{ \pm}=\max \{ \pm u, 0\}$. If $u \in W_{0}^{1, p(z)}(\Omega)$, then $u^{ \pm} \in$ $W_{0}^{1, r(z)}(\Omega), u=u^{+}-u^{-},|u|=u^{+}+u^{-}$. Also, if $u, v \in M(\Omega)$ with $u(z) \leq v(z)$ for a.a. $z \in \Omega$, then we introduce the following order intervals in $W_{0}^{1, p(z)}(\Omega)$ :

$$
\begin{aligned}
& {[u, v]=\left\{h \in W_{0}^{1, p(z)}(\Omega): u(z) \leq h(z) \leq v(z) \text { for a.a. } z \in \Omega\right\},} \\
& {[u)=\left\{h \in W_{0}^{1, p(z)}(\Omega): u(z) \leq h(z) \text { for a.a. } z \in \Omega\right\},} \\
& \text { int }_{C_{0}^{1}(\bar{\Omega})}[u, v]=\text { the interior in } C_{0}^{1}(\bar{\Omega}) \text { of }[u, v] \cap C_{0}^{1}(\bar{\Omega}) .
\end{aligned}
$$

Throughout this work, by $\|\cdot\|$ we denote the norm $W_{0}^{1, p(z)}(\Omega)$. On account of the Poincaré inequality, we have

$$
\|u\|=\|D u\|_{p(z)} \quad \text { for all } u \in W_{0}^{1, p(z)}(\Omega)
$$

Suppose $X$ is a Banach space and $\varphi \in C^{1}(X, \mathbb{R})$. We set

$$
\left.K_{\varphi}=\left\{u \in X: \varphi^{\prime}(u)=0\right\} \quad \text { (the critical set of } \varphi\right)
$$

We say that $\varphi(\cdot)$ satisfies the $C$-condition, if the following property holds:

"If $\left\{u_{n}\right\}_{n \in \mathbb{N}} \subseteq X$ is such that $\left\{\varphi\left(u_{n}\right)\right\}_{n \in \mathbb{N}} \subseteq \mathbb{R}$ is bounded and (1+ $\left.\left\|u_{n}\right\|_{X}\right) \varphi^{\prime}\left(u_{n}\right) \rightarrow 0$ in $X^{*}$ as $n \rightarrow \infty$, then $\left\{u_{n}\right\}_{n \in \mathbb{N}}$ has a strongly convergent subsequence".

Now we will introduce our hypotheses on the perturbation $f(z, x)$.

$\mathrm{H}_{1}: f: \Omega \times \mathbb{R} \rightarrow \mathbb{R}$ is a Carathéodory function such that $f(z, 0)=0$ for a.a. $z \in \Omega$ and

(i) $f(z, x) \leq a(z)\left[1+|x|^{r(z)-1}\right]$ for a.a. $z \in \Omega$, all $x \geq 0$, with $a \in L^{\infty}(\Omega)$, $a(z) \geq 0$ for a.a. $z \in \Omega, r \in C(\bar{\Omega}), p(z)<r(z)<p^{*}(z)$ for all $z \in \bar{\Omega}$; 
(ii) if $F(z, x)=\int_{0}^{x} f(z, s) \mathrm{d} s$, then $\lim _{x \rightarrow+\infty} \frac{F(z, x)}{x^{p_{+}}}=+\infty$ uniformly for a.a. $z \in \Omega$ and there exists $\tau \in C(\bar{\Omega})$ such that

$$
\begin{aligned}
& \tau(z) \in\left(\left(r_{+}-p_{-}\right) \max \left\{\frac{N}{p_{-}}, 1\right\}, p^{*}(z)\right) \text { for all } z \in \bar{\Omega} \\
& 0<\widehat{\delta} \leq \liminf _{x \rightarrow+\infty} \frac{f(z, x) x-p_{+} F(z, x)}{x^{\tau(z)}} \text { uniformly for a.a. } z \in \Omega ;
\end{aligned}
$$

(iii) $\lim _{x \rightarrow 0^{+}} \frac{f(z, x)}{x^{q(z)-1}}=0$ uniformly for a.a. $z \in \Omega$ and we have

$$
0 \leq f(z, x) \text { for a.a. } z \in \Omega \text {, all } x \geq 0
$$

(iv) for every $\rho>0$, we can find $\widehat{\xi}_{\rho}>0$ such that for a.a. $z \in \Omega$, the function

$$
x \mapsto f(z, x)+\widehat{\xi}_{\rho} x^{p(z)-1}
$$

is nondecreasing on $[0, \rho]$.

Remarks Since our goal is to find positive solutions and all the above hypotheses concern the positive semiaxis $\mathbb{R}_{+}=[0,+\infty)$, without any loss of generality, we may assume that $f(z, x)=0$ for a.a. $z \in \Omega$, all $x \leq 0$. Hypothesis $\mathrm{H}_{1}$ (ii) says that for a.a. $z \in \Omega, f(z, \cdot)$ is $\left(p_{+}-1\right)$-superlinear. We do not use the AR-condition, which is common in the literature when dealing with superlinear problems (see, for example, Chang [3], p. 147). Hypothesis $\mathrm{H}_{1}$ (ii) is less restrictive and incorporates in our framework also superlinear perturbations with "slower" growth as $x \rightarrow+\infty$. For example, consider the following function

$$
f(z, x)=\left\{\begin{array}{ll}
\left(x^{+}\right)^{\tau(z)-1}-\left(x^{+}\right)^{s(z)-1} & \text { if } x \leq 1 \\
x^{p_{+}-1} \ln x & \text { if } 1<x
\end{array},\right.
$$

where $\tau, s \in C(\bar{\Omega})$ and $q(z)<\tau(z) \leq s(z)$ for all $z \in \bar{\Omega}$. This function satisfies hypotheses $\mathrm{H}_{1}$ but fails to satisfy the AR-condition. Our hypotheses also incorporate the nonlinearity $f(z, x)=\left(x^{+}\right)^{r(z)-1}$ with $r \in C(\bar{\Omega})$ and $p(z)<r(z)$ for all $z \in \bar{\Omega}$. This is the perturbation used by Byun-Ko [2], where the equation is driven only by the $p(z)$-Laplacian with $p(\cdot)$ satisfying stronger conditions (see hypothesis $\left(p_{M}\right)$ in [2]). Similarly, our hypotheses are more general than those used by Saoudi-Ghanmi [22] (see hypotheses $\left(\mathrm{H}_{3}\right),\left(\mathrm{H}_{4}\right)$ in [22]), who also deal with equations driven by the $p(z)$-Laplacian only. The function $f(z, x)$ given above lies outside the framework provided by the hypotheses in [22]. 


\section{A Purely Singular Problem}

As we already indicated in the Introduction, in order to handle the singular term, we will first consider a purely singular problem. The solution of this problem will allow us to bypass the singularity and deal with $C^{1}$-functionals.

So, in this section, we study the following purely singular problem

$$
\left\{\begin{array}{l}
-\Delta_{p(z)} u(z)-\Delta_{q(z)} u(z)=\lambda u(z)^{-\eta(z)} \text { in } \Omega \\
\left.u\right|_{\partial \Omega}=0, u>0, \lambda>0
\end{array} .\right.
$$

For this problem, we have the following existence and uniqueness result.

Proposition 3.1 If hypotheses $\mathrm{H}_{0}$ hold, then for every $\lambda>0$ problem $\left(\mathrm{Au}_{\lambda}\right)$ has a unique positive solution $\bar{u}_{\lambda} \in$ int $C_{+}$and the map $\stackrel{\circ}{\mathbb{R}}_{+} \ni \lambda \mapsto \bar{u}_{\lambda}$ is nondecreasing and $\left\|\bar{u}_{\lambda}\right\|_{C_{0}^{1}(\bar{\Omega})} \rightarrow 0$ as $\lambda \rightarrow 0^{+}$.

Proof Given $\delta \in(0,1)$, first we solve the following approximation to problem $\left(\mathrm{Au}_{\lambda}\right)$ :

$$
\left\{\begin{array}{l}
-\Delta_{p(z)} u(z)-\Delta_{q(z)} u(z)=\lambda[u(z)+\delta]^{-\eta(z)} \text { in } \Omega \\
\left.u\right|_{\partial \Omega}=0, u>0, \lambda>0
\end{array} .\right.
$$

To solve this problem, we employ a fixed point argument. So, let $g \in C_{0}^{1}(\bar{\Omega})$ and consider the following Dirichlet problem

$$
-\Delta_{p(z)} u(z)-\Delta_{q(z)} u(z)=\lambda[|g(z)|+\delta]^{-\eta(z)} \text { in } \Omega,\left.\quad u\right|_{\partial \Omega}=0 .
$$

The operator $V=A_{p(z)}+A_{q(z)}: W_{0}^{1, p(z)}(\Omega) \rightarrow W^{-1, p^{\prime}(z)}(\Omega)$ is maximal monotone (see Proposition 2.2) and coercive. So $V(\cdot)$ is surjective (see [15], p. 137). Since $\frac{\lambda}{[|g|+\delta]^{\eta(\cdot)}} \in L^{\infty}(\Omega)$, we can find $v_{\delta}^{\lambda} \in W_{0}^{1, p(z)}(\Omega)$ such that

$$
V\left(v_{\delta}^{\lambda}\right)=A_{p(z)}\left(v_{\delta}^{\lambda}\right)+A_{q(z)}\left(v_{\delta}^{\lambda}\right)=\lambda[|g|+\delta]^{-\eta(\cdot)} .
$$

On account of the strict monotonicity of $V(\cdot)$ (see Proposition 2.2), this solution $v_{\delta}^{\lambda}$ is unique. We have

$$
\begin{aligned}
\left\langle V\left(v_{\delta}^{\lambda}\right),-\left(v_{\delta}^{\lambda}\right)^{-}\right\rangle= & \int_{\Omega} \frac{\lambda\left(-v_{\delta}^{\lambda}\right)^{-}}{[|g|+\delta]^{\eta(z)}} \mathrm{d} z \leq 0 \\
& \Rightarrow \rho_{p}\left(D\left(v_{\delta}^{\lambda}\right)^{-}\right) \leq 0 \\
& \Rightarrow v_{\delta}^{\lambda} \geq 0, \quad v_{\delta}^{\lambda} \neq 0 \quad \text { (see Proposition 2.1) }
\end{aligned}
$$

From Theorem 4.1 of Fan-Zhao [6], we have that $v_{\delta}^{\lambda} \in L^{\infty}(\Omega)$. Then Theorem 1.3 of Fan [5] implies that $v_{\delta}^{\lambda} \in C_{+} \backslash\{0\}$. Finally, Proposition 4 of Papageorgiou-QinRădulescu [14] says that $v_{\delta}^{\lambda} \in \operatorname{int} C_{+}$. 
We can define the solution map $\sigma_{\delta}^{\lambda}: C_{0}^{1}(\bar{\Omega}) \rightarrow C_{0}^{1}(\bar{\Omega})$ by setting

$$
\sigma_{\delta}^{\lambda}(g)=v_{\delta}^{\lambda} \in \operatorname{int} C_{+} \cdot
$$

We will show that this map is continuous. To this end, let $g_{n} \rightarrow g$ in $C_{0}^{1}(\bar{\Omega})$ and set $\left(v_{\delta}^{\lambda}\right)_{n}=\sigma_{\delta}^{\lambda}\left(g_{n}\right)$, for all $n \in \mathbb{N}$. We have

$$
\left\langle V\left(\left(v_{\delta}^{\lambda}\right)_{n}\right), h\right\rangle=\int_{\Omega} \frac{\lambda h}{\left[\left|g_{n}\right|+\delta\right]^{\eta(z)}} \mathrm{d} z \quad \text { for all } h \in W_{0}^{1, p(z)}(\Omega) .
$$

In (3.2) we use the test function $\left(v_{\delta}^{\lambda}\right)_{n} \in W_{0}^{1, p(z)}(\Omega)$. We obtain

$$
\begin{gathered}
\rho_{p}\left(D\left(v_{\delta}^{\lambda}\right)_{n}\right)+\rho_{q}\left(D\left(v_{\delta}^{\lambda}\right)_{n}\right) \leq c_{1}\left\|\left(v_{\delta}^{\lambda}\right)_{n}\right\| \text { for some } c_{1}>0, \text { all } n \in \mathbb{N} \\
\Rightarrow\left\{\left(v_{\delta}^{\lambda}\right)_{n}\right\}_{n \in \mathbb{N}} \subseteq W_{0}^{1, p(z)}(\Omega) \text { is bounded (see Proposition 2.1.). }
\end{gathered}
$$

This implies that we can find $c_{2}>0$ such that

$$
\begin{aligned}
& \left(v_{\delta}^{\lambda}\right)_{n} \in L^{\infty}(\Omega) \text { and }\left\|\left(v_{\delta}^{\lambda}\right)_{n}\right\|_{\infty} \leq c_{2} \text { for all } n \in \mathbb{N} \\
& \text { (see [6] and [8], Proposition 3.1). }
\end{aligned}
$$

Then Lemma 3.3 of Fukagai-Narukawa [7] says that there exist $\alpha \in(0,1)$ and $c_{3}>0$ such that

$$
\left(v_{\delta}^{\lambda}\right)_{n} \in C_{0}^{1, \alpha}(\bar{\Omega})=C^{1, \alpha}(\Omega) \cap C_{0}^{1}(\bar{\Omega}), \quad\left\|\left(v_{\delta}^{\lambda}\right)_{n}\right\|_{C_{0}^{1, \alpha}(\Omega)} \leq c_{3}, \quad \text { for all } n \in \mathbb{N}
$$

Recall that $C_{0}^{1, \alpha}(\bar{\Omega}) \hookrightarrow C_{0}^{1}(\bar{\Omega})$ compactly. So, by passing to a suitable subsequence if necessary, we may assume that

$$
\left(v_{\delta}^{\lambda}\right)_{n} \rightarrow v_{\delta}^{\lambda} \text { in } C_{0}^{1}(\bar{\Omega}) \text { as } n \rightarrow \infty .
$$

Then, if we pass to the limit as $n \rightarrow \infty$ in (3.2), we obtain

$$
\begin{aligned}
\left\langle V\left(v_{\delta}^{\lambda}\right), h\right\rangle= & \int_{\Omega} \frac{\lambda h}{[|g|+\delta]^{\eta(z)}} \mathrm{d} z \text { for all } h \in W_{0}^{1, p(z)}(\Omega) \\
& \Rightarrow v_{\delta}^{\lambda}=\sigma_{\delta}^{\lambda}(g) \\
& \Rightarrow \sigma_{\delta}^{\lambda}(\cdot) \text { is continuous. }
\end{aligned}
$$


Also for every $g \in C_{0}^{1}(\bar{\Omega})$ and with $v_{\delta}^{\lambda}=\sigma_{\delta}^{\lambda}(g)$, we have

$$
\begin{aligned}
\left\langle V\left(v_{\delta}^{\lambda}\right), h\right\rangle= & \int_{\Omega} \frac{\lambda h}{[|g|+\delta]^{\eta(z)}} \mathrm{d} z \text { for all } h \in W_{0}^{1, p(z)}(\Omega), \\
& \Rightarrow \rho_{p}\left(D v_{\delta}^{\lambda}\right)+\rho_{q}\left(D v_{\delta}^{\lambda}\right) \leq \frac{1}{\delta^{\eta_{+}}}\left\|v_{\delta}^{\lambda}\right\|, \\
& \Rightarrow \sigma_{\delta}^{\lambda}\left(C_{0}^{1}(\bar{\Omega})\right) \subseteq W_{0}^{1, p(z)}(\Omega) \text { is bounded } \\
& \text { (see Proposition } \left.2.1 \text { and recall that } 1<p_{-}\right) .
\end{aligned}
$$

As above, using (3.4) and the anisotropic regularity theory, we infer that

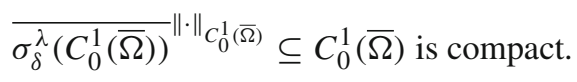

Then, the Schauder-Tychonov fixed point theorem (see [15], p. 298) implies that there exists $\bar{u}_{\delta}^{\lambda} \in C_{0}^{1}(\bar{\Omega})$ such that

$$
\sigma_{\delta}^{\lambda}\left(\bar{u}_{\delta}^{\lambda}\right)=\bar{u}_{\delta}^{\lambda} \in \operatorname{int} C_{+}
$$

This $\bar{u}_{\delta}^{\lambda} \in$ int $C_{+}$is a solution of the approximate problem $\left(\mathrm{Au}_{\lambda}^{\delta}\right)$. We show that this solution is unique. To this end, let $\bar{y}_{\delta}^{\lambda}$ be another solution of $\left(\mathrm{Au}_{\lambda}^{\delta}\right)$. We have

$$
\begin{aligned}
0 \leq & \left\langle V\left(\bar{u}_{\delta}^{\lambda}\right)-V\left(\bar{y}_{\delta}^{\lambda}\right), \bar{u}_{\delta}^{\lambda}-\bar{y}_{\delta}^{\lambda}\right\rangle \\
= & \int_{\Omega} \lambda\left[\frac{1}{\left[\bar{u}_{\delta}^{\lambda}+\delta\right]^{\eta(z)}}-\frac{1}{\left[\bar{y}_{\delta}^{\lambda}+\delta\right]^{\eta(z)}}\right]\left(\bar{u}_{\delta}^{\lambda}-\bar{y}_{\delta}^{\lambda}\right) \mathrm{d} z \leq 0 \\
& \Rightarrow \bar{u}_{\delta}^{\lambda}=\bar{y}_{\delta}^{\lambda} .
\end{aligned}
$$

This proves the uniqueness of the solution $\bar{u}_{\delta}^{\lambda} \in \operatorname{int} C_{+}$of problem $\left(\mathrm{Au}_{\lambda}^{\delta}\right)$.

Claim: $0<\delta^{\prime}<\delta \Rightarrow \bar{u}_{\delta}^{\lambda} \leq \bar{u}_{\delta^{\prime}}^{\lambda}$.

We have

$$
\begin{aligned}
-\Delta_{p(z)} \bar{u}_{\delta^{\prime}}^{\lambda}-\Delta_{q(z)} \bar{u}_{\delta^{\prime}}^{\lambda} & =\lambda\left[\bar{u}_{\delta^{\prime}}^{\lambda}+\delta^{\prime}\right]^{-\eta(z)} \\
& \geq \lambda\left[\bar{u}_{\delta^{\prime}}^{\lambda}+\delta\right]^{-\eta(z)} \quad \text { in } \Omega\left(\text { since } \delta^{\prime}<\delta\right) .
\end{aligned}
$$

We introduce the Carathéodory function $e_{\delta}^{\lambda}: \Omega \times \mathbb{R} \rightarrow \mathbb{R}$ defined by

$$
e_{\delta}^{\lambda}(z, x)= \begin{cases}\lambda\left[x^{+}+\delta\right]^{-\eta(z)} & \text { if } x \leq \bar{u}_{\delta^{\prime}}^{\lambda}(z) \\ \lambda\left[\bar{u}_{\delta^{\prime}}^{\lambda}(z)+\delta\right]^{-\eta(z)} & \text { if } \bar{u}_{\delta^{\prime}}^{\lambda}(z)<x\end{cases}
$$


We set $E_{\delta}^{\lambda}(z, x)=\int_{0}^{x} e_{\delta}^{\lambda}(z, s) \mathrm{d} s$ and consider the $C^{1}$-functional $\gamma_{\lambda}: W_{0}^{1, p(z)}(\Omega)$ $\rightarrow \mathbb{R}$ defined by

$$
\begin{aligned}
& \gamma_{\lambda}(u)=\int_{\Omega} \frac{1}{p(z)}|D u|^{p(z)} \mathrm{d} z+\int_{\Omega} \frac{1}{q(z)}|D u|^{q(z)} \mathrm{d} z-\int_{\Omega} E_{\delta}^{\lambda}(z, u) \mathrm{d} z \\
& \text { for all } u \in W_{0}^{1, p(z)}(\Omega) .
\end{aligned}
$$

We have

$\gamma_{\lambda}(u) \geq \frac{1}{p_{+}} \rho_{p}(D u)-c_{4}$ for some $c_{4}>0$, all $u \in W_{0}^{1, p(z)}(\Omega)($ see (3.6)),

$\Rightarrow \quad \gamma_{\lambda}(\cdot)$ is coercive (see Proposition 2.1 and recall the Poincaré's inequality).

Also the anisotropic Sobolev embedding theorem implies that $\gamma_{\lambda}(\cdot)$ is sequentially weakly lower semicontinuous. So, by the Weierstrass-Tonelli theorem, we can find $\tilde{u}_{\delta}^{\lambda} \in W_{0}^{1, p(z)}(\Omega)$ such that

$$
\begin{aligned}
& \gamma_{\lambda}\left(\tilde{u}_{\delta}^{\lambda}\right)=\min \left[\gamma_{\lambda}(u): u \in W_{0}^{1, p(z)}(\Omega)\right] \\
& \Rightarrow \quad\left\langle\gamma_{\lambda}^{\prime}\left(\widetilde{u}_{\delta}^{\lambda}\right), h\right\rangle=0 \text { for all } h \in W_{0}^{1, p(z)}(\Omega), \\
& \Rightarrow \quad\left\langle A_{p(z)}\left(\tilde{u}_{\delta}^{\lambda}\right), h\right\rangle+\left\langle A_{q(z)}\left(\tilde{u}_{\delta}^{\lambda}\right), h\right\rangle=\int_{\Omega} e_{\delta}^{\lambda}\left(z, \tilde{u}_{\delta}^{\lambda}\right) h \mathrm{~d} z \text { for all } h \in W_{0}^{1, p(z)}(\Omega)
\end{aligned}
$$

In (3.7) we choose the test function $h=-\left(\widetilde{u}_{\delta}^{\lambda}\right)^{-} \in W_{0}^{1, p(z)}(\Omega)$. We obtain

$$
\begin{gathered}
\rho_{p}\left(D\left(\tilde{u}_{\delta}^{\lambda}\right)^{-}\right)+\rho_{q}\left(D\left(\tilde{u}_{\delta}^{\lambda}\right)^{-}\right)=0, \\
\Rightarrow \quad \tilde{u}_{\delta}^{\lambda} \geq 0, \quad \tilde{u}_{\delta}^{\lambda} \neq 0 .
\end{gathered}
$$

Next in (3.7) we choose $h=\left[\widetilde{u}_{\delta}^{\lambda}-\bar{u}_{\delta^{\prime}}^{\lambda}\right]^{+} \in W_{0}^{1, p(z)}(\Omega)$. We have

$$
\begin{aligned}
& \left\langle A_{p(z)}\left(\tilde{u}_{\delta}^{\lambda}\right),\left(\tilde{u}_{\delta}^{\lambda}-\bar{u}_{\delta^{\prime}}^{\lambda}\right)^{+}\right\rangle+\left\langle A_{q(z)}\left(\tilde{u}_{\delta}^{\lambda}\right),\left(\tilde{u}_{\delta}^{\lambda}-\bar{u}_{\delta^{\prime}}^{\lambda}\right)^{+}\right\rangle \\
& =\int_{\Omega} \lambda\left[\bar{u}_{\delta^{\prime}}^{\lambda}+\delta\right]^{-\eta(z)}\left(\tilde{u}_{\delta}^{\lambda}-\bar{u}_{\delta^{\prime}}^{\lambda}\right)^{+} \mathrm{d} z \quad(\text { see }(3.6)) \\
& \leq\left\langle A_{p(z)}\left(\bar{u}_{\delta^{\prime}}^{\lambda}\right),\left(\tilde{u}_{\delta}^{\lambda}-\bar{u}_{\delta^{\prime}}^{\lambda}\right)^{+}\right\rangle+\left\langle A_{q(z)}\left(\bar{u}_{\delta^{\prime}}^{\lambda}\right),\left(\tilde{u}_{\delta}^{\lambda}-\bar{u}_{\delta^{\prime}}^{\lambda}\right)^{+}\right\rangle \quad(\text { see (3.5)) } \\
& \quad \Rightarrow \tilde{u}_{\delta}^{\lambda} \leq \bar{u}_{\delta^{\prime}}^{\lambda} .
\end{aligned}
$$

So, we have proved that

$$
\begin{aligned}
& \tilde{u}_{\delta}^{\lambda} \in\left[0, \bar{u}_{\delta^{\prime}}^{\lambda}\right], \quad \tilde{u}_{\delta}^{\lambda} \neq 0 \\
& \quad \Rightarrow \quad \tilde{u}_{\delta}^{\lambda}=\bar{u}_{\delta}^{\lambda} \in \operatorname{int} C_{+} \quad(\operatorname{see}(3.8),(3.6),(3.7)) \\
& \Rightarrow \quad \bar{u}_{\delta}^{\lambda} \leq \bar{u}_{\delta^{\prime}}^{\lambda} .
\end{aligned}
$$


This proves the claim.

Next we let $\delta \rightarrow 0^{+}$to produce a solution of the auxiliary problem $\left(\mathrm{Au}_{\lambda}\right)$. Let $\delta_{n} \rightarrow 0^{+}$and set $\bar{u}_{n}^{\lambda}=\bar{u}_{\delta_{n}}^{\lambda} \in$ int $C_{+}$, for all $n \in \mathbb{N}$. We have

$$
\begin{aligned}
& 0 \leq \bar{u}_{1}^{\lambda} \leq \bar{u}_{n}^{\lambda} \text { for all } n \in \mathbb{N} \text { (see the Claim), } \\
& \left\langle A_{p(z)}\left(\bar{u}_{n}^{\lambda}\right), h\right\rangle+\left\langle A_{q(z)}\left(\bar{u}_{n}^{\lambda}\right), h\right\rangle=\int_{\Omega} \lambda\left[\bar{u}_{n}^{\lambda}+\delta_{n}\right]^{-\eta(z)} h \mathrm{~d} z \\
& \quad \text { for all } h \in W_{0}^{1, p(z)}(\Omega), \text { all } n \in \mathbb{N} .
\end{aligned}
$$

In (3.10) we use the test function $\bar{u}_{n}^{\lambda} \in W_{0}^{1, p(z)}(\Omega)$. We obtain

$$
\begin{aligned}
\rho_{p}\left(D \bar{u}_{n}^{\lambda}\right)+\rho_{q}\left(D \bar{u}_{n}^{\lambda}\right) \leq & \int_{\Omega} \frac{\lambda \bar{u}_{n}^{\lambda}}{\left(\bar{u}_{n}^{\lambda}\right)^{\eta(z)}} \mathrm{d} z=\int_{\Omega} \lambda\left(\bar{u}_{n}^{\lambda}\right)^{1-\eta(z)} \mathrm{d} z \\
& \Rightarrow \quad \rho_{p}\left(D \bar{u}_{n}^{\lambda}\right) \leq \lambda c_{5}\left\|\bar{u}_{n}^{\lambda}\right\| \text { for some } c_{5}>0, \text { all } n \in \mathbb{N} \\
& \Rightarrow\left\{\bar{u}_{n}^{\lambda}\right\}_{n \in \mathbb{N}} \subseteq W_{0}^{1, p(z)}(\Omega) \text { is bounded } \\
& \text { (see Proposition } \left.2.1 \text { and recall that } 1<p_{-}\right)
\end{aligned}
$$

On account of (3.11), we may assume that

$$
\begin{aligned}
& \bar{u}_{n}^{\lambda} \stackrel{w}{\rightarrow} \bar{u}_{\lambda} \text { in } W_{0}^{1, p(z)}(\Omega) \text { and } \\
& \left|D \bar{u}_{n}^{\lambda}\right|^{p(z)-2} D \bar{u}_{n}^{\lambda}+\left|D \bar{u}_{n}^{\lambda}\right|^{q(z)-2} D \bar{u}_{n}^{\lambda} \stackrel{w}{\rightarrow} \zeta \text { in } L^{p^{\prime}(z)}\left(\Omega, \mathbb{R}^{N}\right) .
\end{aligned}
$$

From (3.9) we have

$$
0 \leq \frac{1}{\left[\bar{u}_{n}^{\lambda}+\delta_{n}\right]^{\eta(z)}} \leq \frac{1}{\left(\bar{u}_{1}^{\lambda}\right)^{\eta(z)}}
$$

Then, for every $h \in C_{c}^{\infty}(\Omega)$, we have that $\bar{u}_{1}^{\lambda}(z) \geq c_{h}>0$ for all $z \in \operatorname{supp} h$ (recall that $\bar{u}_{1}^{\lambda} \in$ int $\left.C_{+}\right)$. Hence, for $h \in C_{c}^{\infty}(\Omega)$, we have

$$
0 \leq \frac{|h|}{\left[\bar{u}_{n}^{\lambda}+\delta_{n}\right]^{\eta(z)}} \leq c_{6}(h)|h| \text { for some } c_{6}(h)>0 \text {, all } n \in \mathbb{N}
$$

So, if in (3.10) we pass to the limit as $n \rightarrow \infty$ and use the dominated convergence theorem [see (3.12)], we obtain

$$
\langle-\operatorname{div} \zeta, h\rangle=\int_{\Omega} \frac{h}{\bar{u}_{\lambda}^{\eta(z)}} \mathrm{d} z \text { for all } h \in C_{c}^{\infty}(\Omega) .
$$

The density of $C_{c}^{\infty}(\Omega)$ in $W_{0}^{1, p(z)}(\Omega)$ implies that (3.13) holds for all $h \in$ $W_{0}^{1, p(z)}(\Omega)$ and so $-\operatorname{div} \zeta=\frac{1}{\bar{u}_{\lambda}^{\eta(\cdot)}}$. 
As above, we have

$$
\left|\left\langle\frac{1}{\left[\bar{u}_{n}^{\lambda}+\delta_{n}\right]^{\eta(\cdot)}}-\frac{1}{\bar{u}_{\lambda}^{\eta(\cdot)}}, h\right\rangle\right| \leq \lambda \epsilon_{n}\|h\| \text { for all } h \in C_{c}^{\infty}(\Omega) \text {, with } \epsilon_{n} \rightarrow 0^{+} .
$$

Exploiting the density of $C_{c}^{\infty}(\Omega)$ in $W_{0}^{1, p(z)}(\Omega)$, we infer that (3.14) holds for all $h \in W_{0}^{1, p(z)}(\Omega)$. It follows that

$$
\frac{1}{\left[\bar{u}_{n}^{\lambda}+\delta_{n}\right]^{\eta(\cdot)}} \rightarrow \frac{1}{\bar{u}_{\lambda}^{\eta(\cdot)}} \text { in } W^{-1, p^{\prime}(z)}(\Omega)
$$

From (3.11), (3.15) and Theorem 2.1 of Boccardo-Murat [1], we have that $D \bar{u}_{n}^{\lambda}(z) \rightarrow$ $D \bar{u}_{\lambda}(z)$ for a.a. $z \in \Omega$. It follows that

$$
\zeta=\left|D \bar{u}_{\lambda}\right|^{p(z)-2} D \bar{u}_{\lambda}+\left|D \bar{u}_{\lambda}\right|^{q(z)-2} D \bar{u}_{\lambda}
$$

and then

$$
\left\langle A_{p(z)}\left(\bar{u}_{\lambda}\right), h\right\rangle+\left\langle A_{q(z)}\left(\bar{u}_{\lambda}\right), h\right\rangle=\int_{\Omega} \frac{\lambda h}{\bar{u}_{\lambda}^{\eta(z)}} \text { for all } h \in W_{0}^{1, p(z)}(\Omega) .
$$

Since $\bar{u}_{1}^{\lambda} \leq \bar{u}_{\lambda}$ (see (3.9), (3.12)), we infer that $\bar{u}_{\lambda}$ is a positive solution of $\left(\mathrm{Au}_{\lambda}\right)$. Then, from Theorem B.1 of Saoudi-Ghanmi [22] (see also Giacomoni-SchindlerTakáč [9], Theorem B.1), we have that $\bar{u}_{\lambda} \in \operatorname{int} C_{+}$. As before, we check that $\bar{u}_{\lambda} \in$ int $C_{+}$is the unique positive solution of $\left(\mathrm{Au}_{\lambda}\right)$.

Next we check that $\lambda \mapsto \bar{u}_{\lambda}$ is nondecreasing from $\stackrel{\circ}{\mathbb{R}}_{+}=(0, \infty)$ into int $C_{+}$. So, let $0<\lambda<\lambda^{\prime}$. We have

$$
-\Delta_{p(z)} \bar{u}_{\lambda^{\prime}}-\Delta_{q(z)} \bar{u}_{\lambda^{\prime}}=\lambda^{\prime} \bar{u}_{\lambda^{\prime}}^{-\eta(z)} \geq \lambda \bar{u}_{\lambda^{\prime}}^{-\eta(z)}
$$

We consider the Carathéodory function $\widehat{g}_{\lambda}: \Omega \times \stackrel{\circ}{\mathbb{R}}_{+} \rightarrow \stackrel{\circ}{\mathbb{R}}_{+}$defined by

$$
\widehat{g}_{\lambda}(z, x)= \begin{cases}\lambda x^{-\eta(z)} & \text { if } 0<x \leq \bar{u}_{\lambda^{\prime}}(z) \\ \lambda \bar{u}_{\lambda^{\prime}}(z)^{-\eta(z)} & \text { if } \bar{u}_{\lambda^{\prime}}(z)<x\end{cases}
$$

Note that from the proof of the Lemma of Lazer-McKenna [13], we have

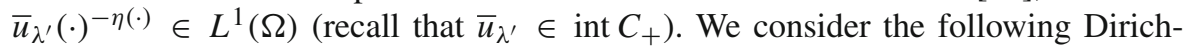
let problem

$$
-\Delta_{p(z)} u(z)-\Delta_{q(z)} u(z)=\widehat{g}_{\lambda}(z, u(z)) \text { in } \Omega,\left.\quad u\right|_{\partial \Omega}=0, \quad u>0
$$

Reasoning as in the first part of the proof (using approximations of this problem), we show that it has a unique solution $\widetilde{u}_{\lambda} \in$ int $C_{+}$and $\widetilde{u}_{\lambda} \leq \bar{u}_{\lambda^{\prime}}$. We conclude that $\tilde{u}_{\lambda}=\bar{u}_{\lambda}[\operatorname{see}(3.16)]$ and so $\bar{u}_{\lambda} \leq \bar{u}_{\lambda^{\prime}}$. 
Finally let $\lambda_{n} \rightarrow 0^{+}$and let $\bar{u}_{n}=\bar{u}_{\lambda_{n}} \in \operatorname{int} C_{+}, n \in \mathbb{N}$, be the unique solution of $\left(\mathrm{P}_{\lambda_{n}}\right)$ produced earlier. We have

$\left\langle A_{p(z)}\left(\bar{u}_{n}\right), h\right\rangle+\left\langle A_{q(z)}\left(\bar{u}_{n}\right), h\right\rangle=\int_{\Omega} \lambda_{n} \bar{u}_{n}^{-\eta(z)} h \mathrm{~d} z$ for all $h \in W_{0}^{1, p(z)}(\Omega)$, all $n \in \mathbb{N}$.

Using the test function $h=\bar{u}_{n} \in W_{0}^{1, p(z)}(\Omega)$ we obtain

$$
\begin{aligned}
& \rho_{p}\left(D \bar{u}_{n}\right)+\rho_{q}\left(D \bar{u}_{n}\right) \leq \lambda_{n} c_{7}\left\|u_{n}\right\| \text { for some } c_{7}>0, \text { all } n \in \mathbb{N} \\
& \left.\quad \Rightarrow \quad \bar{u}_{n} \rightarrow 0 \text { in } W_{0}^{1, p(z)}(\Omega) \text { (see Proposition } 2.1\right)
\end{aligned}
$$

Then, the anisotropic regularity theory (see [22]), implies that $\left\{\bar{u}_{n}\right\}_{n \in \mathbb{N}} \subseteq C_{0}^{1}(\bar{\Omega})$ is relatively compact. Therefore $\bar{u}_{n} \rightarrow 0$ in $C_{0}^{1}(\bar{\Omega})$ [see (3.17)].

\section{Positive Solutions}

We introduce the following two sets

$$
\begin{aligned}
& \mathscr{L}=\left\{\lambda>0: \text { problem }\left(\mathrm{P}_{\lambda}\right) \text { has a positive solution }\right\} \\
& S_{\lambda}=\text { set of positive solutions of problem }\left(\mathrm{P}_{\lambda}\right)
\end{aligned}
$$

Proposition 4.1 If hypotheses $\mathrm{H}_{0}, \mathrm{H}_{1}$ hold, then $\mathscr{L} \neq \varnothing$ and for every $\lambda>0, S_{\lambda} \subseteq$ int $C_{+}$.

Proof Hypotheses $\mathrm{H}_{1}$ (i), (iii) imply that given $\epsilon>0$, we can find $c_{8}=c_{8}(\epsilon)>0$ such that for $\widehat{r} \in C(\bar{\Omega})$ with $\widehat{r}>r, \widehat{r}_{-}>p_{+}$, we have

$$
\begin{aligned}
& f(z, x) \leq \epsilon x^{q(z)-1}+c_{8} x^{\widehat{r}(z)-1} \text { for a.a. } z \in \Omega, \text { all } x \geq 0, \\
& \quad \Rightarrow \quad F(z, x) \leq \frac{\epsilon}{p(z)} x^{q(z)}+\frac{c_{8}}{r(z)} x^{\widehat{r}(z)} \text { for a.a. } z \in \Omega \text {, all } x \geq 0 .
\end{aligned}
$$

Let $\bar{u}_{\lambda} \in$ int $C_{+}$be the unique solution of the auxiliary problem $\left(\mathrm{Au}_{\lambda}\right)$. Recall that $\bar{u}_{\lambda}(\cdot)^{-\eta(\cdot)} \in L^{1}(\Omega)$. We introduce the Carathéodory function $k_{\lambda}: \Omega \times \mathbb{R} \rightarrow \mathbb{R}$ defined by

$$
k_{\lambda}(z, x)= \begin{cases}\lambda \bar{u}_{\lambda}(z)^{-\eta(z)}+f\left(z, x^{+}\right) & \text {if } x \leq \bar{u}_{\lambda}(z) \\ \lambda x^{-\eta(z)}+f(z, x) & \text { if } \bar{u}_{\lambda}(z)<x\end{cases}
$$


We set $K_{\lambda}(z, x)=\int_{0}^{x} k_{\lambda}(z, s) \mathrm{d} s$ and consider the $C^{1}$-functional $\psi_{\lambda}: W_{0}^{1, p(z)}(\Omega)$ $\rightarrow \mathbb{R}$ defined by

$$
\begin{aligned}
\psi_{\lambda}(u)= & \int_{\Omega} \frac{1}{p(z)}|D u|^{p(z)} \mathrm{d} z+\int_{\Omega} \frac{1}{q(z)}|D u|^{q(z)} \mathrm{d} z \\
& -\int_{\Omega} K_{\lambda}(z, u) \mathrm{dz}, \quad \text { for all } u \in W_{0}^{1, p(z)}(\Omega)
\end{aligned}
$$

Using (4.1) and (4.2), we have

$$
\begin{aligned}
\psi_{\lambda}(u) \geq & \frac{1}{p_{+}} \rho_{p}(D u)-\int_{\left\{0 \leq u \leq \bar{u}_{\lambda}\right\}} \lambda u \bar{u}_{\lambda}^{-\eta(z)} \mathrm{d} z- \\
& \int_{\left\{\bar{u}_{\lambda}<u\right\}} \frac{\lambda}{1-\eta(z)}\left[u^{1-\eta(z)}-\bar{u}_{\lambda}^{1-\eta(z)}\right] \mathrm{d} z-\frac{\epsilon}{q_{-}} \rho_{q}(u)-\frac{c_{8}}{\widehat{r}_{-}} \rho_{\widehat{r}}(u) .
\end{aligned}
$$

Let $\widehat{d}(\cdot)=d(\cdot, \partial \Omega)$. From Lemma 14.16, p. 355, of Gilbarg-Trudinger [10], we know that we can find $\delta_{0}>0$ such that $\widehat{d} \in C^{2}\left(\Omega_{\delta_{0}}\right)$ where $\Omega_{\delta_{0}}=\{z \in \bar{\Omega}: \widehat{d}(z)<$ $\left.\delta_{0}\right\}$. It follows that $\widehat{d} \in C_{+} \backslash\{0\}$. Since $\bar{u}_{\lambda} \in$ int $C_{+}$, on account of Proposition 4.1.22, p. 274, of Papageorgiou-Rădulescu-Repovš [15], we can find $c_{9}>0$ such that

$$
c_{9} \widehat{d} \leq \bar{u}_{\lambda}
$$

Then we have

$$
\begin{aligned}
\int_{\Omega} \frac{|u|}{\bar{u}_{\lambda}^{\eta(z)}} \mathrm{d} z & =\int_{\Omega} \bar{u}_{\lambda}^{1-\eta(z)} \frac{|u|}{\bar{u}_{\lambda}} \mathrm{d} z \\
& \leq c_{10} \int_{\Omega} \frac{|u|}{\widehat{d}} \mathrm{~d} z \text { for some } c_{10}>0(\text { see }(4.4)) \\
& \leq c_{11}\|u\| \text { for some } c_{11}>0
\end{aligned}
$$

where in the last inequality, we have used the anisotropic Hardy inequality due to Harjuletho-Hästö-Koskenoja [11]. Returning to (4.3) and using (4.5), for all $u \in$ $W_{0}^{1, p(z)}(\Omega)$ with $\|u\|=\rho<1$ we have

$$
\psi_{\lambda} \geq \frac{1}{p_{+}} \rho^{p_{+}}-c_{12} \rho^{\widehat{r}_{-}}-c_{13}\left[\lambda \rho+\epsilon \rho^{q_{-}}\right] \text {for some } c_{12}, c_{13}>0 .
$$

Since $p_{+}<\widehat{r}_{-}$, choosing $\rho \in(0,1)$ small, we have

$$
\frac{1}{p_{+}} \rho^{p_{+}}-c_{12} \rho^{\widehat{r}_{-}}>0
$$

Then choosing $\lambda>0$ and $\epsilon>0$ small, from (4.6) we see that

$$
\psi_{\lambda}(u) \geq m_{\lambda}>0 \text { for all } u \in W_{0}^{1, p(z)}(\Omega),\|u\|=\rho .
$$


Let $v \in$ int $C_{+}$. Since $\bar{u}_{\lambda} \in$ int $C_{+}$, we can find $t \in(0,1)$ small such that $t v \leq \bar{u}_{\lambda}$ (see [15], p. 274). We have

$$
\begin{array}{r}
\psi_{\lambda}(t v) \leq \frac{t^{q_{-}}}{q_{-}}\left[\rho_{p}(D v)+\rho_{q}(D v)\right]-\lambda t \int_{\Omega} \frac{v}{\bar{u}_{\lambda}^{\eta(z)}} \mathrm{d} z \\
\quad\left(\text { see }(4.2) \text { and recall that } F \geq 0, q_{-}<p_{-}\right) .
\end{array}
$$

We have seen above that $\frac{v}{\bar{u}_{\lambda}^{\eta(\cdot)}} \in L^{1}(\Omega)$. Hence

$$
\psi_{\lambda}(t v) \leq c_{14} t^{q}-c_{15} t \text { for some } c_{14}, c_{15}>0
$$

Since $q_{-}>1$, choosing $t \in(0,1)$ even smaller if necessary, we have

$$
\psi_{\lambda}(t v)<0 \text { and }\|t v\| \leq \rho .
$$

Consider the closed ball

$$
\bar{B}_{\rho}=\left\{u \in W_{0}^{1, p(z)}(\Omega):\|u\| \leq \rho\right\}
$$

The reflexivity of $W_{0}^{1, p(z)}(\Omega)$ and the Eberlein-Smulian theorem imply that $\bar{B}_{\rho}$ is sequentially weakly compact. Also, using the anisotropic Sobolev embedding theorem, we check that $\psi_{\lambda}(\cdot)$ is sequentially weakly compact. So, we can find $u_{\lambda} \in W_{0}^{1, p(z)}(\Omega)$ such that

$$
\begin{aligned}
\psi_{\lambda}\left(u_{\lambda}\right)= & \min \left[\psi_{\lambda}(u): u \in \bar{B}_{\rho}\right] \\
& \Rightarrow \psi_{\lambda}\left(u_{\lambda}\right)<0=\psi_{\lambda}(0) \quad(\operatorname{see}(4.8)) \\
& \Rightarrow u_{\lambda} \neq 0
\end{aligned}
$$

From (4.7), we see that

$$
0<\left\|u_{\lambda}\right\|<\rho .
$$

Then, (4.9) and (4.10) imply that

$$
\begin{aligned}
& \psi_{\lambda}^{\prime}\left(u_{\lambda}\right)=0 \\
& \quad \Rightarrow\left\langle A_{p(z)}\left(u_{\lambda}\right), h\right\rangle+\left\langle A_{q(z)}\left(u_{\lambda}\right), h\right\rangle=\int_{\Omega} k_{\lambda}\left(z, u_{\lambda}\right) h \mathrm{~d} z \text { for all } h \in W_{0}^{1, p(z)}(\Omega) .
\end{aligned}
$$


In (4.11) we use the test function $h=\left(\bar{u}_{\lambda}-u_{\lambda}\right)^{+} \in W_{0}^{1, p(z)}(\Omega)$. Then

$$
\begin{aligned}
& \left\langle A_{p(z)}\left(u_{\lambda}\right),\left(\bar{u}_{\lambda}-u_{\lambda}\right)^{+}\right\rangle+\left\langle A_{q(z)}\left(u_{\lambda}\right),\left(\bar{u}_{\lambda}-u_{\lambda}\right)^{+}\right\rangle \\
& \quad=\int_{\Omega}\left[\lambda \bar{u}_{\lambda}^{-\eta(z)}+f\left(z, u_{\lambda}^{+}\right)\right]\left(\bar{u}_{\lambda}-u_{\lambda}\right)^{+} \mathrm{d} z \quad(\text { see }(4.2) .) \\
& \quad \geq \int_{\Omega} \lambda \bar{u}_{\lambda}^{-\eta(z)}\left(\bar{u}_{\lambda}-u_{\lambda}\right)^{+} \mathrm{d} z \quad(\text { since } f \geq 0) \\
& =\left\langle A_{p(z)}\left(\bar{u}_{\lambda}\right),\left(\bar{u}_{\lambda}-u_{\lambda}\right)^{+}\right\rangle+\left\langle A_{q(z)}\left(\bar{u}_{\lambda}\right),\left(\bar{u}_{\lambda}-u_{\lambda}\right)^{+}\right\rangle \quad \text { (see Proposition 3.1) } \\
& \quad \Rightarrow \bar{u}_{\lambda} \leq u_{\lambda} .
\end{aligned}
$$

Then (4.12), (4.2) and (4.11) imply that

$$
\begin{gathered}
u_{\lambda} \in S_{\lambda} \quad(\lambda>0 \text { small }) \\
\Rightarrow \quad \mathscr{L} \neq \varnothing .
\end{gathered}
$$

Moreover, the anisotropic regularity theory (see Theorem B.1 of [22]) and the anisotropic maximum principle (see Proposition 4 of [14]) imply that $S_{\lambda} \subseteq$ int $C_{+}$for all $\lambda \in \mathscr{L}$.

The next proposition shows that $\mathscr{L}$ is connected.

Proposition 4.2 If hypotheses $\mathrm{H}_{0}, \mathrm{H}_{1}$ hold, $\lambda \in \mathscr{L}$ and $\mu \in(0, \lambda)$, then $\mu \in \mathscr{L}$.

Proof Since $\lambda \in \mathscr{L}$, we can find $u_{\lambda} \in S_{\lambda} \subseteq$ int $C_{+}$(see Proposition 4.1). Recall that $u_{\lambda}^{-\eta(\cdot)} \in L^{1}(\Omega)$. Also we have $\bar{u}_{\mu} \leq \bar{u}_{\lambda} \leq u_{\lambda}$ (see Proposition 3.1 and use the fact that $f \geq 0$ ). We introduce the Carathéodory function $\vartheta_{\mu}: \Omega \times \mathbb{R} \rightarrow \mathbb{R}$ defined by

$$
\vartheta_{\mu}(z, x)= \begin{cases}\mu \bar{u}_{\mu}(z)^{-\eta(z)}+f\left(z, u_{\mu}(z)\right) & \text { if } x<\bar{u}_{\mu}(z) \\ \mu x^{-\eta(z)}+f(z, x) & \text { if } \bar{u}_{\mu}(z) \leq x \leq u_{\lambda}(z) \\ \mu u_{\lambda}(z)^{-\eta(z)}+f\left(z, u_{\lambda}(z)\right) & \text { if } u_{\lambda}(z)<x\end{cases}
$$

We set $\Theta_{\mu}(z, x)=\int_{0}^{x} \vartheta_{\mu}(z, s) \mathrm{d} s$ and consider the $C^{1}$-functional $w_{\mu}: W_{0}^{1, p(z)}$ $(\Omega) \rightarrow \mathbb{R}$ defined by

$$
\begin{aligned}
& w_{\mu}(u)=\int_{\Omega} \frac{1}{p(z)}|D u|^{p(z)} \mathrm{d} z+\int_{\Omega} \frac{1}{q(z)}|D u|^{q(z)} \mathrm{d} z-\int_{\Omega} \Theta_{\mu}(z, u) \mathrm{d} z \\
& \text { for all } u \in W_{0}^{1, p(z)}(\Omega) .
\end{aligned}
$$

From (4.13), it is clear that $w_{\mu}(\cdot)$ is coercive. Also, it is sequentially weakly lower semicontinuous. So, we can find $u_{\mu} \in W_{0}^{1, p(z)}(\Omega)$ such that

$$
w_{\mu}\left(u_{\mu}\right)=\min \left[w_{\mu}(u): u \in W_{0}^{1, p(z)}(\Omega)\right] .
$$


From (4.14) we have

$$
\left\langle A_{p(z)}\left(u_{\mu}\right), h\right\rangle+\left\langle A_{q(z)}\left(u_{\mu}\right), h\right\rangle=\int_{\Omega} \vartheta_{\mu}\left(z, u_{\mu}\right) h \mathrm{~d} z \quad \text { for all } h \in W_{0}^{1, p(z)}(\Omega) .
$$

In (4.15) first we use the test function $h=\left(\bar{u}_{\mu}-u_{\mu}\right)^{+} \in W_{0}^{1, p(z)}(\Omega)$. Then

$$
\begin{aligned}
& \left\langle A_{p(z)}\left(u_{\mu}\right),\left(\bar{u}_{\mu}-u_{\mu}\right)^{+}\right\rangle+\left\langle A_{q(z)}\left(u_{\mu}\right),\left(\bar{u}_{\mu}-u_{\mu}\right)^{+}\right\rangle \\
& =\int_{\Omega}\left[\mu \bar{u}_{\mu}^{-\eta(z)}+f\left(z, \bar{u}_{\mu}\right)\right]\left(\bar{u}_{\mu}-u_{\mu}\right)^{+} \mathrm{d} z \quad(\text { see }(4.13)) \\
& \geq \int_{\Omega} \mu \bar{u}_{\mu}^{-\eta(z)}\left(\bar{u}_{\mu}-u_{\mu}\right)^{+} \mathrm{d} z \quad(\text { since } f \geq 0) \\
& =\left\langle A_{p(z)}\left(\bar{u}_{\mu}\right),\left(\bar{u}_{\mu}-u_{\mu}\right)^{+}\right\rangle+\left\langle A_{q(z)}\left(\bar{u}_{\mu}\right),\left(\bar{u}_{\mu}-u_{\mu}\right)^{+}\right\rangle \\
& \quad \Rightarrow \bar{u}_{\mu} \leq u_{\mu} .
\end{aligned}
$$

Next in (4.15) we choose $h=\left(u_{\mu}-u_{\lambda}\right)^{+} \in W_{0}^{1, p(z)}(\Omega)$. We have

$$
\begin{aligned}
& \left\langle A_{p(z)}\left(u_{\mu}\right),\left(u_{\mu}-u_{\lambda}\right)^{+}\right\rangle+\left\langle A_{q(z)}\left(u_{\mu}\right),\left(u_{\mu}-u_{\lambda}\right)^{+}\right\rangle \\
& \quad=\int_{\Omega}\left[\mu u_{\lambda}^{-\eta(z)}+f\left(z, u_{\lambda}\right)\right]\left(u_{\mu}-u_{\lambda}\right)^{+} \mathrm{d} z \quad(\text { see }(4.13)) \\
& \quad \leq \int_{\Omega}\left[\lambda u_{\lambda}^{-\eta(z)}+f\left(z, u_{\lambda}\right)\right]\left(u_{\mu}-u_{\lambda}\right)^{+} \mathrm{d} z \quad(\text { since } \mu<\lambda) \\
& =\left\langle A_{p(z)}\left(u_{\lambda}\right),\left(u_{\mu}-u_{\lambda}\right)^{+}\right\rangle+\left\langle A_{q(z)}\left(u_{\lambda}\right),\left(u_{\mu}-u_{\lambda}\right)^{+}\right\rangle \quad\left(\text { since } u_{\lambda} \in S_{\lambda}\right) \\
& \quad \Rightarrow u_{\mu} \leq u_{\lambda} .
\end{aligned}
$$

So, we have proved that

$$
u_{\mu} \in\left[\bar{u}_{\mu}, u_{\lambda}\right]
$$

From (4.16), (4.13) and (4.15), we conclude that

$$
\begin{gathered}
u_{\mu} \in S_{\mu} \subseteq \operatorname{int} C_{+}, \\
\Rightarrow \quad \mu \in \mathscr{L} .
\end{gathered}
$$

A by-product of the above proof is the following weak monotonicity property for the solution multifunction $\lambda \mapsto S_{\lambda}$.

Corollary 4.3 If hypotheses $\mathrm{H}_{0}, \mathrm{H}_{1}$ hold, $\lambda \in \mathscr{L}, u_{\lambda} \in S_{\lambda} \subseteq$ int $C_{+}$and $\mu \in(0, \lambda)$, then $\mu \in \mathscr{L}$ and we can find $u_{\mu} \in S_{\mu} \subseteq$ int $C_{+}$such that $u_{\mu} \leq u_{\lambda}$.

Using Proposition 2.3, we can improve the assertion of this corollary. 
Proposition 4.4 If hypotheses $\mathrm{H}_{0}, \mathrm{H}_{1}$ hold, $\lambda \in \mathscr{L}, u_{\lambda} \in S_{\lambda} \subseteq$ int $C_{+}$and $\mu \in(0, \lambda)$, then $\mu \in \mathscr{L}$ and we can find $u_{\mu} \in S_{\mu} \subseteq$ int $C_{+}$such that

$$
u_{\lambda}-u_{\mu} \in \operatorname{int} C_{+}
$$

Proof From Corollary 4.3, we already know that $\mu \in \mathscr{L}$ and there exists $u_{\mu} \in S_{\mu} \subseteq$ int $C_{+}$such that

$$
u_{\mu} \leq u_{\lambda}
$$

Let $\rho=\left\|u_{\lambda}\right\|_{\infty}$ and let $\widehat{\xi}_{\rho}>0$ be as postulated by hypothesis $\mathrm{H}_{1}(\mathrm{iv})$. We have

$$
\begin{aligned}
- & \Delta_{p(z)} u_{\mu}-\Delta_{q(z)} u_{\mu}+\widehat{\xi}_{\rho} u_{\mu}^{p(z)-1}-\lambda u_{\mu}^{-\eta(z)} \\
& =f\left(z, u_{\mu}\right)+\widehat{\xi}_{\rho} u_{\mu}^{p(z)-1}-(\lambda-\mu) u_{\mu}^{-\eta(z)} \\
& \leq f\left(z, u_{\lambda}\right)+\widehat{\xi}_{\rho} u_{\lambda}^{p(z)-1} \quad\left(\text { see }(4.17), \text { hypothesis } \mathrm{H}_{1}(\mathrm{iv}) \text { and use that } \mu<\lambda\right) \\
& =-\Delta_{p(z)} u_{\lambda}-\Delta_{q(z)} u_{\lambda}+\widehat{\xi}_{\rho} u_{\lambda}^{p(z)-1}-\lambda u_{\lambda}^{-\eta(z)} \quad\left(\text { since } u_{\lambda} \in S_{\lambda}\right) .
\end{aligned}
$$

Since $u_{\mu} \in$ int $C_{+}$, we have

$$
0 \preceq(\lambda-\mu) u_{\mu}^{-\eta(\cdot)} .
$$

So, from (4.18) and Proposition 2.3, it follows that

$$
u_{\lambda}-u_{\mu} \in \operatorname{int} C_{+}
$$

Let $\lambda^{*}=\sup \mathscr{L}$.

Proposition 4.5 If hypotheses $\mathrm{H}_{0}, \mathrm{H}_{1}$ hold, then $\lambda^{*}<\infty$.

Proof On account of hypotheses $\mathrm{H}_{1}(\mathrm{i})$, (ii), (iii), we can find $\lambda_{0}>0$ such that

$$
\lambda_{0} x^{-\eta(z)}+f(z, x) \geq x^{p(z)-1} \text { for a.a. } z \in \Omega, \text { all } x \geq 0 .
$$

Let $\lambda>\lambda_{0}$ and suppose that $\lambda \in \mathscr{L}$. Then we can find $u_{\lambda} \in S_{\lambda} \subseteq$ int $C_{+}$. Consider

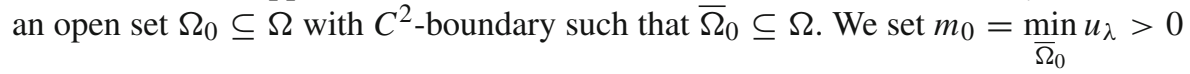


(since $u_{\lambda} \in$ int $C_{+}$). For $\delta>0$, set $m_{0}^{\delta}=m_{0}+\delta$, let $\rho=\left\|u_{\lambda}\right\|_{\infty}$ and let $\widehat{\xi}_{\rho}>0$ be as postulated by hypothesis $\mathrm{H}_{1}$ (iv). We have

$$
\begin{aligned}
- & \Delta_{p(z)} m_{0}^{\delta}+\widehat{\xi}_{\rho}\left(m_{0}^{\delta}\right)^{p(z)-1}-\lambda\left(m_{0}^{\delta}\right)^{-\eta(z)} \\
& \leq \widehat{\xi}_{\rho} m_{0}^{p(z)-1}+\chi(\delta)-\frac{\lambda}{m_{0}^{\eta(z)}+\delta \eta(z)} \\
& \text { with } \chi(\delta) \rightarrow 0^{+} \text {as } \delta \rightarrow 0^{+}(\text {recall that } 0<\eta(z)<1 \text { for all } z \in \bar{\Omega}) \\
\leq & {\left[\widehat{\xi}_{\rho}+1\right] m_{0}^{p(z)-1}+\chi(\delta)+\frac{\lambda \delta^{\eta(z)}}{m_{0}^{2 \eta(z)}}-\frac{\lambda}{m_{0}^{\eta(z)}} } \\
= & m_{0}^{p(z)-1}-\lambda_{0} m_{0}^{-\eta(z)}+\widehat{\xi}_{\rho} m_{0}^{p(z)-1}+\widehat{\chi}(\delta)-\frac{\lambda-\lambda_{0}}{m_{0}^{\eta(z)}} \quad \text { with } \widehat{\chi}(\delta) \rightarrow 0^{+} \text {as } \delta \rightarrow 0^{+} \\
\leq & \left.f\left(z, m_{0}\right)+\widehat{\xi}_{\rho} m_{0}^{p(z)-1} \text { for } \delta>0 \text { small (see }(4.19) \text { and recall that } \lambda>\lambda_{0}\right) \\
\leq & f\left(z, u_{\lambda}\right)+\widehat{\xi}_{\rho} u_{\lambda}^{p(z)-1} \quad\left(\text { see hypothesis } \mathrm{H}_{1}(\text { iv })\right) \\
= & -\Delta_{p(z)} u_{\lambda}-\Delta_{q(z)} u_{\lambda}+\widehat{\xi}_{\rho} u_{\lambda}^{p(z)-1} \text { in } \Omega_{0} .
\end{aligned}
$$

Invoking Proposition 6 of Papageorgiou-Rădulescu-Repovš [18], we infer that

$$
m_{0}^{\delta}<u_{\lambda}(z) \text { for all } z \in \Omega_{0} \text {, all } \delta \in(0,1) \text { small, }
$$

a contradiction to the definition of $m_{0}$.

Therefore $\lambda \notin \mathscr{L}$ and so $\lambda^{*} \leq \lambda_{0}<\infty$.

Next we show that $\lambda \in\left(0, \lambda^{*}\right)$ we have multiplicity of positive solutions for problem $\left(\mathrm{P}_{\lambda}\right)$.

Proposition 4.6 If hypotheses $\mathrm{H}_{0}, \mathrm{H}_{1}$ hold and $\lambda \in\left(0, \lambda^{*}\right)$, then problem $\left(\mathrm{P}_{\lambda}\right)$ has at least two positive solutions

$$
u_{0}, \widehat{u} \in \operatorname{int} C_{+} .
$$

Proof Let $0<\mu<\lambda<\delta<\lambda^{*}$. We know that $\mu, \delta \in \mathscr{L}$ and on account of Proposition 4.4, we can find $u_{\delta} \in S_{\delta} \subseteq$ int $C_{+}, u_{0} \in S_{\lambda} \subseteq$ int $C_{+}$and $u_{\mu} \in S_{\mu} \subseteq$ int $C_{+}$such that

$$
\begin{gathered}
u_{\delta}-u_{0} \in \operatorname{int} C_{+} \text {and } u_{0}-u_{\mu} \in \operatorname{int} C_{+} \\
\Rightarrow \quad u_{0} \in \operatorname{int}_{C_{0}^{1}(\bar{\Omega})}\left[u_{\mu}, u_{\delta}\right] .
\end{gathered}
$$

Recall that $u_{\mu}(\cdot)^{-\eta(\cdot)}, u_{\delta}(\cdot)^{-\eta(\cdot)} \in L^{1}(\Omega)$. We introduce the Carathéodory function $\widehat{\beta}_{\lambda}: \Omega \times \mathbb{R} \rightarrow \mathbb{R}$ defined by

$$
\widehat{\beta}_{\lambda}(z, x)= \begin{cases}\lambda u_{\mu}(z)^{-\eta(z)}+f\left(z, u_{\mu}(z)\right) & \text { if } x<u_{\mu}(z) \\ \lambda x^{-\eta(z)}+f(z, x) & \text { if } u_{\mu}(z) \leq x \leq u_{\delta}(z) \\ \lambda u_{\delta}(z)^{-\eta(z)}+f\left(z, u_{\delta}(z)\right) & \text { if } u_{\delta}(z)<x\end{cases}
$$


We set $\widehat{B}_{\lambda}(z, x)=\int_{0}^{x} \widehat{\beta}_{\lambda}(z, s) \mathrm{d} s$ and consider the $C^{1}$-functional $\widehat{\zeta}_{\lambda}: W_{0}^{1, p(z)}(\Omega)$ $\rightarrow \mathbb{R}$ defined by

$$
\begin{aligned}
& \widehat{\zeta}_{\lambda}(u)=\int_{\Omega} \frac{1}{p(z)}|D u|^{p(z)} \mathrm{d} z+\int_{\Omega} \frac{1}{q(z)}|D u|^{q(z)} \mathrm{d} z-\int_{\Omega} \widehat{B}_{\lambda}(z, u) \mathrm{d} z \\
& \quad \text { for all } u \in W_{0}^{1, p(z)}(\Omega) .
\end{aligned}
$$

We have

$$
\begin{aligned}
\widehat{\zeta}_{\lambda}(u) & \geq \frac{1}{p_{+}} \rho(D u)-c_{16} \text { for some } c_{16}>0, \text { all } u \in W_{0}^{1, p(z)}(\Omega)(\text { see }(4.21)), \\
& \Rightarrow \widehat{\zeta}_{\lambda}(\cdot) \text { is coercive (see Proposition 2.1). }
\end{aligned}
$$

Also $\widehat{\zeta}_{\lambda}(\cdot)$ is sequentially weakly lower semicontinuous. Therefore, we can find $\widehat{u}_{0} \in W_{0}^{1, p(z)}(\Omega)$ such that

$$
\widehat{\zeta}_{\lambda}\left(\widehat{u}_{0}\right)=\min \left[\widehat{\zeta}_{\lambda}(u): u \in W_{0}^{1, p(z)}(\Omega)\right]
$$

From (4.21) and the anisotropic regularity theory, we have

$$
K_{\widehat{\zeta}_{\lambda}} \subseteq\left[u_{\mu}, u_{\delta}\right] \cap \operatorname{int} C_{+} \cdot
$$

From (4.22), (4.21) and (4.20), we see that

$$
u_{0}, \widehat{u}_{0} \in K_{\widehat{\zeta}_{\lambda}}
$$

If $u_{0} \neq \widehat{u}_{0}$, then we already have two positive smooth solutions of $\left(\mathrm{P}_{\lambda}\right)$ and so we are done. Thus we may assume that

$$
u_{0}=\widehat{u}_{0} .
$$

We consider the Carathéodory function $\beta_{\lambda}: \Omega \times \mathbb{R} \rightarrow \mathbb{R}$ defined by

$$
\beta_{\lambda}(z, x)=\left\{\begin{array}{ll}
\lambda u_{\mu}(z)^{-\eta(z)}+f\left(z, u_{\mu}(z)\right) & \text { if } x \leq u_{\mu}(z) \\
\lambda x^{-\eta(z)}+f(z, x) & \text { if } u_{\mu}(z)<x
\end{array} .\right.
$$

We set $B_{\lambda}(z, x)=\int_{0}^{x} \beta_{\lambda}(z, s) \mathrm{d} s$ and consider the $C^{1}$-functional $\zeta_{\lambda}: W_{0}^{1, p(z)}(\Omega)$ $\rightarrow \mathbb{R}$ defined by

$$
\begin{aligned}
& \zeta_{\lambda}(u)=\int_{\Omega} \frac{1}{p(z)}|D u|^{p(z)} \mathrm{d} z+\int_{\Omega} \frac{1}{q(z)}|D u|^{q(z)} \mathrm{d} z-\int_{\Omega} B_{\lambda}(z, u) \mathrm{d} z \\
& \quad \text { for all } u \in W_{0}^{1, p(z)}(\Omega) .
\end{aligned}
$$


From (4.21) and (4.25), we see that

$$
\left.\zeta_{\lambda}\right|_{\left[0, u_{\delta}\right]}=\left.\widehat{\zeta}_{\lambda}\right|_{\left[0, u_{\delta}\right]}
$$

Combining (4.24), (4.22), (4.20) and (4.26), we see that

$$
\begin{aligned}
& u_{0} \text { is a local } C_{0}^{1}(\bar{\Omega}) \text {-minimizer of } \zeta_{\lambda}(\cdot) \\
& \quad \Rightarrow \quad u_{0} \text { is a local } W_{0}^{1, p(z)}(\Omega) \text {-minimizer of } \zeta_{\lambda}(\cdot) \\
& \text { (see Gasiński-Papageorgiou [8], Proposition 3.3). }
\end{aligned}
$$

From (4.25), it follows that $K_{\zeta_{\lambda}} \subseteq\left[u_{\mu}\right) \cap$ int $C_{+}$. So, we may assume that $K_{\zeta_{\lambda}}$ is finite or otherwise we already have an infinity of positive smooth solutions of $\left(\mathrm{P}_{\lambda}\right)$ and so we are done. Then, from (4.27) and using Theorem 5.7.6, p. 449, of [15], we can find $\rho \in(0,1)$ small such that

$$
\zeta_{\lambda}\left(u_{0}\right)<\inf \left[\zeta_{\lambda}(u):\left\|u-u_{0}\right\|=\rho\right]=m_{\lambda} .
$$

If $u \in \operatorname{int} C_{+}$, then on account of hypothesis $\mathrm{H}_{1}(\mathrm{ii})$, we have

$$
\zeta_{\lambda}(t v) \rightarrow-\infty \text { as } t \rightarrow+\infty
$$

Moreover, Proposition 4.1, of Gasiński-Papageorgiou [8], implies that

$$
\zeta_{\lambda}(\cdot) \text { satisfies the } \mathrm{C} \text {-condition. }
$$

Then (4.28), (4.29) and (4.30) permit the use of the mountain pass theorem. So, we can find $\widehat{u} \in W_{0}^{1, p(z)}(\Omega)$ such that

$$
\begin{aligned}
\widehat{u} \in K_{\zeta_{\lambda}} \subseteq\left[u_{\mu}\right) \cap \operatorname{int} C_{+}, \quad \zeta_{\lambda}\left(u_{0}\right)<m_{\lambda} \leq \zeta_{\lambda}(\widehat{u}), \\
\quad \Rightarrow \widehat{u} \in \operatorname{int} C_{+} \text {is a positive solution of }\left(\mathrm{P}_{\lambda}\right), \widehat{u} \neq u_{0} .
\end{aligned}
$$

We produce a lower bound for the elements of $S_{\lambda}$. This will be helpful in proving the admissibility of the critical parameter $\lambda^{*}$ and also in producing the minimal positive solution for every $\lambda \in \mathscr{L}$ (see Sect. 5).

Proposition 4.7 If hypotheses $\mathrm{H}_{0}, \mathrm{H}_{1}$ hold and $\lambda \in \mathscr{L}$, then $\bar{u}_{\lambda} \leq u$ for all $u \in S_{\lambda}$.

Proof Let $u \in S_{\lambda} \subseteq$ int $C_{+}$and consider the Carathéodory function $\sigma: \Omega \times \stackrel{\circ}{\mathbb{R}}_{+} \rightarrow \stackrel{\circ}{\mathbb{R}}_{+}$ defined by

$$
\sigma(z, x)= \begin{cases}x^{-\eta(z)} & \text { if } 0<x \leq u(z) \\ u(z)^{-\eta(z)} & \text { if } u(z)<x\end{cases}
$$


We consider the following Dirichlet problem

$$
\left\{\begin{array}{l}
-\Delta_{p(z)} u(z)-\Delta_{q(z)} u(z)=\lambda \sigma(z, u(z)) \text { in } \Omega \\
\left.u\right|_{\partial \Omega}=0, u>0, \lambda>0
\end{array} .\right.
$$

As in the proof of Proposition 3.1, via approximations and fixed point theory, we show that problem $\left(\mathrm{Au}_{\lambda}^{\prime}\right)$ as a unique positive solution

$$
\tilde{u}_{\lambda} \in[0, u] \cap \operatorname{int} C_{+} \quad(\operatorname{see}(4.31)) .
$$

From Proposition 3.1, it follows that

$$
\begin{aligned}
& \tilde{u}_{\lambda}=\bar{u}_{\lambda}, \\
& \quad \Rightarrow \quad \bar{u}_{\lambda} \leq u \text { for all } u \in S_{\lambda} .
\end{aligned}
$$

Using this bound, we can prove the admissibility of $\lambda^{*}$.

Proposition 4.8 If hypotheses $\mathrm{H}_{0}, \mathrm{H}_{1}$ hold, then $\lambda^{*} \in \mathscr{L}$.

Proof Let $\left\{\lambda_{n}\right\}_{n \in \mathbb{N}} \subseteq \mathscr{L}$ such that $\lambda_{n} \uparrow \lambda^{*}$. From Proposition 3.1, we know that $\bar{u}_{\lambda_{1}} \leq$ $\bar{u}_{\lambda_{n}}$ for all $n \in \mathbb{N}$. Also let $\tilde{u}_{n+1} \in S_{\lambda_{n+1}} \subseteq$ int $C_{+}$. On account of Proposition 4.7, we have $\bar{u}_{\lambda_{1}} \leq \widetilde{u}_{n+1}$. Then we can introduce the Carathéodory function $\widehat{l_{n}}: \Omega \times \mathbb{R} \rightarrow \mathbb{R}$ defined by

$$
\widehat{l}_{n}(z, x)= \begin{cases}\lambda_{n} \bar{u}_{\lambda_{1}}(z)^{-\eta(z)}+f\left(z, x^{+}\right) & \text {if } x<\bar{u}_{\lambda_{1}}(z) \\ \lambda_{n} x^{-\eta(z)}+f(z, x) & \text { if } \bar{u}_{\lambda_{1}}(z) \leq x \leq \widetilde{u}_{n+1}(z) . \\ \lambda_{n} \widetilde{u}_{n+1}(z)+f\left(z, \widetilde{u}_{n+1}(z)\right) & \text { if } \widetilde{u}_{n+1}(z)<x\end{cases}
$$

We set $\widehat{L}_{n}(z, x)=\int_{0}^{x} \widehat{l}_{n}(z, s) \mathrm{d} s$ and consider the $C^{1}$-functional $\widehat{\chi}_{n}: W_{0}^{1, p(z)}(\Omega) \rightarrow$ $\mathbb{R}$ defined by

$$
\begin{aligned}
& \widehat{\chi}_{n}(u)=\int_{\Omega} \frac{1}{p(z)}|D u|^{p(z)} \mathrm{d} z+\int_{\Omega} \frac{1}{q(z)}|D u|^{q(z)} \mathrm{d} z-\int_{\Omega} \widehat{L}_{n}(z, u) \mathrm{d} z \\
& \quad \text { for all } u \in W_{0}^{1, p(z)}(\Omega) .
\end{aligned}
$$

From (4.32), we see that $\widehat{\chi}_{n}(\cdot)$ is coercive. Also it is sequentially weakly lower semicontinuous. Hence we can find $u_{n} \in W_{0}^{1, p(z)}(\Omega)$ such that

$$
\begin{aligned}
& \widehat{\chi}_{n}\left(u_{n}\right)=\min \left[\widehat{\chi}_{n}(u): u \in W_{0}^{1, p(z)}(\Omega)\right], \\
& \quad \Rightarrow \quad\left\langle\widehat{\chi}_{n}^{\prime}\left(u_{n}\right), h\right\rangle=0 \text { for all } h \in W_{0}^{1, p(z)}(\Omega) .
\end{aligned}
$$


If in (4.33) we use first the test function $h=\left(\bar{u}_{\lambda_{1}}-u_{n}\right)^{+} \in W_{0}^{1, p(z)}(\Omega)$ and then the test function $h=\left(u_{n}-\widetilde{u}_{n+1}\right)^{+} \in W_{0}^{1, p(z)}(\Omega)$, then using (4.32) we obtain

$$
\begin{aligned}
& u_{n} \in\left[\bar{u}_{\lambda_{1}}, \widetilde{u}_{n+1}\right], \\
& \quad \Rightarrow \quad u_{n} \in S_{\lambda_{n}} \subseteq \operatorname{int} C_{+} \quad(\operatorname{see}(4.32) .) .
\end{aligned}
$$

Let $l_{n}: \Omega \times \mathbb{R} \rightarrow \mathbb{R}$ be the Carathéodory function defined by

$$
l_{n}(z, x)=\left\{\begin{array}{ll}
\lambda_{n} \bar{u}_{\lambda_{1}}(z)^{-\eta(z)}+f\left(z, x^{+}\right) & \text {if } x \leq \bar{u}_{\lambda_{1}}(z) \\
\lambda_{n} x^{-\eta(z)}+f(z, x) & \text { if } \bar{u}_{\lambda_{1}}(z)<x
\end{array} .\right.
$$

We set $L_{n}(z, x)=\int_{0}^{x} l_{n}(z, s) \mathrm{d} s$ and consider the $C^{1}$-functional $\chi_{n}: W_{0}^{1, p(z)}(\Omega) \rightarrow$ $\mathbb{R}$ defined by

$$
\begin{aligned}
& \chi_{n}(u)=\int_{\Omega} \frac{1}{p(z)}|D u|^{p(z)} \mathrm{d} z+\int_{\Omega} \frac{1}{q(z)}|D u|^{q(z)} \mathrm{d} z-\int_{\Omega} L_{n}(z, u) \mathrm{d} z \\
& \text { for all } u \in W_{0}^{1, p(z)}(\Omega) .
\end{aligned}
$$

From (4.32) and (4.34), we see that

$$
\left.\chi_{n}\right|_{\left[0, \tilde{u}_{n+1}\right]}=\left.\widehat{\chi}_{n}\right|_{\left[0, \tilde{u}_{n+1}\right]},\left.\quad \chi_{n}^{\prime}\right|_{\left[0, \widetilde{u}_{n+1}\right]}=\left.\widehat{\chi}_{n}^{\prime}\right|_{\left[0, \tilde{u}_{n+1}\right]} .
$$

Also we have

$$
\begin{aligned}
\widehat{\chi}_{n}\left(u_{n}\right) \leq \widehat{\chi_{n}}\left(\bar{u}_{\lambda_{1}}\right) \leq & \left.\frac{1}{q_{-}}\left[\rho_{p}\left(D \bar{u}_{\lambda_{1}}\right)+\rho_{q}\left(D \bar{u}_{\lambda_{1}}\right)\right]-\int_{\Omega} \lambda_{n} \bar{u}_{\lambda_{1}}^{1-\eta(z)} \mathrm{d} z \quad \text { (since } f \geq 0\right) \\
\leq & \left.\frac{1}{q_{-}}\left[\rho_{p}\left(D \bar{u}_{\lambda_{1}}\right)+\rho_{q}\left(D \bar{u}_{\lambda_{1}}\right)\right]-\int_{\Omega} \lambda_{1} \bar{u}_{\lambda_{1}}^{1-\eta(z)} \mathrm{d} z \quad \text { (since } \lambda_{1} \leq \lambda_{n}\right) \\
\leq & \frac{1}{q_{-}}\left[\rho_{p}\left(D \bar{u}_{\lambda_{1}}\right)+\rho_{q}\left(D \bar{u}_{\lambda_{1}}\right)\right]-\left[\rho_{p}\left(D \bar{u}_{\lambda_{1}}\right)+\rho_{q}\left(D \bar{u}_{\lambda_{1}}\right)\right] \\
& (\text { see Proposition 3.1) } \\
& <0 \quad\left(\text { since } 1<q_{-}\right) .
\end{aligned}
$$

Then, from (4.35) we have

$$
\chi_{n}^{\prime}\left(u_{n}\right)=0 \text { and } \chi_{n}\left(u_{n}\right)<0 \text { for all } n \in \mathbb{N} .
$$

As in Proposition 4.1 of Gasiński-Papageorgiou [8], from (4.36) we deduce that

$$
\begin{aligned}
& u_{n} \rightarrow u_{*} \text { in } W_{0}^{1, p(z)}(\Omega) \\
& \Rightarrow \quad\left\langle A_{p(z)}\left(u_{*}\right), h\right\rangle+\left\langle A_{q(z)}\left(u_{*}\right), h\right\rangle=\int_{\Omega}\left[\lambda^{*} u_{*}^{-\eta(z)}+f\left(z, u_{*}\right)\right] h \mathrm{~d} z \\
& \quad \text { for all } h \in W_{0}^{1, p(z)}(\Omega)
\end{aligned}
$$


and

$$
\bar{u}_{\lambda_{1}} \leq u_{*}
$$

Therefore $u_{*} \in S_{\lambda^{*}} \subseteq$ int $C_{+}$and so $\lambda^{*} \in \mathscr{L}$.

So, we have proved that

$$
\mathscr{L}=\left(0, \lambda^{*}\right]
$$

We can state the following bifurcation-type theorem about the positive solutions of problem $\left(\mathrm{P}_{\lambda}\right)$.

Theorem 4.9 If hypotheses $\mathrm{H}_{0}, \mathrm{H}_{1}$ hold, then there exists $\lambda^{*}>0$ such that

(a) for all $\lambda \in\left(0, \lambda^{*}\right)$ problem $\left(\mathrm{P}_{\lambda}\right)$ has at least two positive solutions $u_{0}, \widehat{u} \in \operatorname{int} C_{+}$, $u_{0} \neq \widehat{u}$;

(b) for $\lambda=\lambda^{*}$ problem $\left(\mathrm{P}_{\lambda}\right)$ has at least one positive solution $u_{*} \in \operatorname{int} C_{+}$;

(c) for all $\lambda>\lambda^{*}$ problem $\left(\mathrm{P}_{\lambda}\right)$ has no positive solutions.

\section{Minimal Positive Solution}

In this section, we show that for every $\lambda \in \mathscr{L}=\left(0, \lambda^{*}\right]$ problem $\left(\mathrm{P}_{\lambda}\right)$ has a smallest positive solution $u_{\lambda}^{*} \in$ int $C_{+}$(minimal - or barrier - positive solution) and we prove the monotonicity and continuity properties of the map $\lambda \mapsto u_{\lambda}^{*}$.

Proposition 5.1 If hypotheses $\mathrm{H}_{0}, \mathrm{H}_{1}$ hold and $\lambda \in \mathscr{L}=\left(0, \lambda^{*}\right]$, then problem $\left(\mathrm{P}_{\lambda}\right)$ has a smallest positive solution $u_{\lambda}^{*} \in$ int $C_{+}$(that is, $u_{\lambda}^{*} \leq u$ for all $u \in S_{\lambda}$ ).

Proof From Papageorgiou-Rădulescu-Repovš [16] (see the proof of Proposition 7) we have that $S_{\lambda}$ is downward directed (that is, if $u_{1}, u_{2} \in S_{\lambda}$, then we can find $u \in S_{\lambda}$ such that $u \leq u_{1}, u \leq u_{2}$ ). Invoking Lemma 3.10, p. 178, of Hu-Papageorgiou [12], we can find a decreasing sequence $\left\{u_{n}\right\}_{n \in \mathbb{N}} \subseteq S_{\lambda} \subseteq$ int $C_{+}$such that

$$
\inf S_{\lambda}=\inf _{n \in \mathbb{N}} u_{n} .
$$

We have

$$
\begin{aligned}
& \bar{u}_{\lambda} \leq u_{n} \leq u_{1} \text { for all } n \in \mathbb{N}(\text { see Proposition 4.7), } \\
& \left\langle A_{p(z)}\left(u_{n}\right), h\right\rangle+\left\langle A_{q(z)}\left(u_{n}\right), h\right\rangle=\int_{\Omega}\left[\lambda u_{n}^{-\eta(z)}+f\left(z, u_{n}\right)\right] h \mathrm{~d} z \\
& \text { for all } h \in W_{0}^{1, p(z)}(\Omega), \text { all } n \in \mathbb{N} .
\end{aligned}
$$

From (5.1) and (5.2), it follows that

$$
\left\{u_{n}\right\}_{n \in \mathbb{N}} \subseteq W_{0}^{1, p(z)}(\Omega) \text { is bounded. }
$$


From (5.3) and Proposition 2.2 (in particular the $(S)_{+}$-property), we obtain

$$
\begin{aligned}
u_{n} & \rightarrow u_{\lambda}^{*} \text { in } W_{0}^{1, p(z)}(\Omega) \\
& \Rightarrow\left\langle A_{p(z)}\left(u_{\lambda}^{*}\right), h\right\rangle+\left\langle A_{q(z)}\left(u_{\lambda}^{*}\right), h\right\rangle \\
& =\int_{\Omega}\left[\lambda\left(u_{\lambda}^{*}\right)^{-\eta(z)}+f\left(z, u_{\lambda}^{*}\right)\right] h \mathrm{~d} z \text { for all } h \in W_{0}^{1, p(z)}(\Omega),
\end{aligned}
$$

and

$$
\bar{u}_{\lambda} \leq u_{\lambda}^{*}
$$

Therefore we conclude that

$$
u_{\lambda}^{*} \in S_{\lambda} \subseteq \operatorname{int} C_{+} \text {and } u_{\lambda}^{*}=\inf S_{\lambda} .
$$

We consider the map $\lambda \mapsto u_{\lambda}^{*}$ from $\mathscr{L}=\left(0, \lambda^{*}\right]$ into int $C_{+}$. We will say that this map is strictly increasing if $0<\lambda<\lambda^{\prime} \leq \lambda^{*}$, then $u_{\lambda^{\prime}}^{*}-u_{\lambda}^{*} \in \operatorname{int} C_{+}$.

Proposition 5.2 If hypotheses $\mathrm{H}_{0}, \mathrm{H}_{1}$ hold, then minimal solution map $\lambda \mapsto u_{\lambda}^{*}$ is

(a) strictly increasing;

(b) left continuous.

Proof (a) Let $0<\lambda<\lambda^{\prime} \leq \lambda^{*}$. From Proposition 4.4, we know that we can find $u_{\lambda} \in S_{\lambda} \subseteq$ int $C_{+}$such that $u_{\lambda^{\prime}}^{*}-u_{\lambda} \in$ int $C_{+}$. Since $u_{\lambda}^{*} \leq u_{\lambda}$, it follows that $u_{\lambda^{\prime}}^{*}-u_{\lambda}^{*} \in \operatorname{int} C_{+}$and this proves that the minimal solution map is strictly increasing.

(b) Let $\left\{\lambda_{n}\right\}_{n \in \mathbb{N}} \subseteq \mathscr{L}=\left(0, \lambda^{*}\right]$ such that $\lambda_{n} \rightarrow \lambda^{-}$. We have

$$
\begin{aligned}
& u_{\lambda_{1}}^{*} \leq u_{\lambda_{n}}^{*} \leq u_{\lambda}^{*} \text { for all } n \in \mathbb{N}(\text { see }(\mathrm{a})), \\
& \Rightarrow \quad\left\{u_{\lambda_{n}}^{*}\right\}_{n \in \mathbb{N}} \subseteq W_{0}^{1, p(z)}(\Omega)
\end{aligned}
$$

From (5.4) and the anisotropic regularity theory (see [22]), we have that $\left\{u_{\lambda_{n}}^{*}\right\}_{n \in \mathbb{N}} \subseteq C_{0}^{1, \alpha}(\bar{\Omega})$ is bounded with $\alpha \in(0,1)$ and $C_{0}^{1, \alpha}(\bar{\Omega})=C^{1, \alpha}(\bar{\Omega}) \cap$ $C_{0}^{1}(\bar{\Omega})$. From the compact embedding of $C_{0}^{1, \alpha}(\bar{\Omega})$ into $C_{0}^{1}(\bar{\Omega})$, it follows that we may assume that at least for a subsequence we have

$$
u_{\lambda_{n}}^{*} \rightarrow \tilde{u}_{\lambda} \text { in } C_{0}^{1}(\bar{\Omega}) .
$$

Suppose $\tilde{u}_{\lambda} \neq u_{\lambda}^{*}$. Then we can find $z_{0} \in \Omega$ such that

$$
\begin{aligned}
u_{\lambda}^{*}\left(z_{0}\right) & <\tilde{u}_{\lambda}\left(z_{0}\right), \\
\Rightarrow & u_{\lambda}^{*}\left(z_{0}\right)<u_{\lambda_{n}}^{*}\left(z_{0}\right) \text { for all } n \geq n_{0},
\end{aligned}
$$


which contradicts (a). So, $\tilde{u}_{\lambda}=u_{\lambda}^{*}$ and by Urysohn's criterion for the convergence of sequences, we infer that for the initial sequence we have

$$
\begin{aligned}
u_{\lambda_{n}}^{*} & \rightarrow u_{\lambda}^{*} \text { in } C_{0}^{1}(\bar{\Omega}) \\
& \Rightarrow \lambda \rightarrow u_{\lambda}^{*} \text { is left continuous. }
\end{aligned}
$$

So, we can state the following theorem for the minimal positive solutions of problem $\left(\mathrm{P}_{\lambda}\right)$.

Theorem 5.3 If hypotheses $\mathrm{H}_{0}, \mathrm{H}_{1}$ hold, then for every $\lambda \in \mathscr{L}=\left(0, \lambda^{*}\right]$ problem $\left(\mathrm{P}_{\lambda}\right)$ has a smallest positive solution (minimal positive solution) $u_{\lambda}^{*} \in \operatorname{int} C_{+}$and the minimal solution map $\lambda \mapsto u_{\lambda}^{*}$ from $\mathscr{L}$ into int $C_{+}$is strictly increasing and left continuous.

Open Access This article is licensed under a Creative Commons Attribution 4.0 International License, which permits use, sharing, adaptation, distribution and reproduction in any medium or format, as long as you give appropriate credit to the original author(s) and the source, provide a link to the Creative Commons licence, and indicate if changes were made. The images or other third party material in this article are included in the article's Creative Commons licence, unless indicated otherwise in a credit line to the material. If material is not included in the article's Creative Commons licence and your intended use is not permitted by statutory regulation or exceeds the permitted use, you will need to obtain permission directly from the copyright holder. To view a copy of this licence, visit http://creativecommons.org/licenses/by/4.0/.

\section{References}

1. Boccardo, L., Murat, F.: Almost everywhere convergence of the gradients of solutions to elliptic and parabolic equations. Nonlinear Anal. 19, 581-597 (1992)

2. Byun, S.S., Ko, E.: Global $C^{1, \alpha}$ regularity and existence of multiple solutions for singular $p(x)$ Laplacian equations. Calc. Var. Partial Differ. Equ. 56, 76 (2017)

3. Chang, K.C.: Infinite Dimensional Morse Theory and Multiple Solution Problems. Birkhäuser, Boston (1993)

4. Diening, L., Harjulehto, P., Hästö, P., Růžička, M.: Lebesgue and Sobolev Spaces with Variable Exponent. Lecture Notes in Mathematics, vol. 2017. Springer, Heidelberg (2011)

5. Fan, X.: Global $C^{1, \alpha}$-regularity for variable exponent elliptic equations in divergence form. J. Differ. Equ. 235, 397-417 (2007)

6. Fan, X., Zhao, D.: A class of De Giorgi type and Hölder continuity. Nonlinear Anal. Theory Methods Appl. 36, 295-318 (1999)

7. Fukagai, N., Narukawa, K.: On the existence of multiple positive solutions of quasilinear elliptic eigenvalue problems. Ann. Mat. Pura Appl. 186, 539-564 (2007)

8. Gasiński, L., Papageorgiou, N.S.: Anisotropic nonlinear Neumann problems. Calc. Var. Partial Differ. Equ. 42, 323-354 (2011)

9. Giacomoni, J., Schindler, I., Takáč, P.: Sobolev versus Hölder local minimizers and existence of multiple solutions for a singular quasilinear equation. Ann. Scuola Norm. Sup. Pisa Cl. Sci. 6, 117-158 (2007)

10. Gilbarg, D., Trudinger, N.S.: Elliptic Partial Differential Equations of Second Order, 2nd edn. Springer, Berlin (1998)

11. Harjulehto, P., Hästö, P., Koskenoja, M.: Hardy’s inequality in a variable exponent Sobolev space. Georgian Math. J. 12, 431-442 (2005)

12. Hu, S., Papageorgiou, N.S.: Handbook of Multivalued Analysis. Volume I: Theory. Kluwer Academic Publishers, Dordrecht (1997) 
13. Lazer, A.C., McKenna, P.J.: On a singular nonlinear elliptic boundary value problem. Proc. Am. Math. Soc. 111, 721-730 (1991)

14. Papageorgiou, N.S., Qin, D., Rădulescu, V.D.: Anisotropic double-phase problems with indefinite potential: multiplicity of solutions. Anal. Math. Phys. 10, 63 (2020)

15. Papageorgiou, N.S., Rădulescu, V.D., Repov̌̌, D.D.: Nonlinear Analysis-Theory and Methods. Springer, Switzerland (2019)

16. Papageorgiou, N.S., Rădulescu, V.D., Repovš, D.D.: Positive solutions for perturbations of the Robin eigenvalue problem plus an indefinite potential. Discrete Contin. Dyn. Syst. 37, 2589-2615 (2017)

17. Papageorgiou, N.S., Rădulescu, V.D., Repovš, D.D.: Anisotropic equations with indefinite potential and competing nonlinearities. Nonlinear Anal. Theory Methods Appl. 201, 111861 (2020)

18. Papageorgiou, N.S., Rădulescu, V.D., Repovš, D.D.: Nonlinear nonhomogeneous singular problems. Calc. Var. Partial Differ. Equ. 59, 9 (2020)

19. Rădulescu, V.D., Repovš, D.D.: Partial Differential Equations with Variable Exponents: Variational Methods and Qualitative Analysis. CRC Press, Boca Raton (2015)

20. Repovš, D.D., Saoudi, K.: The Nehari manifold approach for singular equations involving the $p(x)$ Laplace operator. Complex Var. Elliptic Equ. (2021). (in press)

21. Saoudi, K., Kratou, M., Alsadhan, S.: Multiplicity results for the $p(x)$-Laplacian equation with singular nonlinearities and nonlinear Neumann boundary condition. Int. J. Differ. Equ. 2016, 3149482 (2016)

22. Saoudi, K., Ghanmi, A.: A multiplicity result for a singular equation involving the $p(x)$-Laplace operator. Complex Var. Elliptic. Equ. 62, 695-725 (2017)

23. Saoudi, K.: The fibering map approach to a $p(x)$-Laplacian equation with singular nonlinearities and nonlinear Neumann boundary conditions. Rocky Mt. J. Math. 48(3), 927-946 (2018)

Publisher's Note Springer Nature remains neutral with regard to jurisdictional claims in published maps and institutional affiliations. 